\title{
REDUCIBILITY OF POLYNOMIALS IN SEVERAL VARIABLES. II
}

\author{
A. SCHINZEL \\ In Loving Memory of Ernst G. Straus
}

\begin{abstract}
Let $f_{i}\left(x_{i}\right)$ be non-constant rational functions over a field $K(i=$ $1,2, \ldots, n)$. A necessary and sufficient condition is given for reducibility over $K$ of the numerator of the sum $\sum_{i=1}^{n} f_{l}\left(x_{l}\right)$ in its reduced form, provided $n \geq 3$. In particular the numerator is irreducible if char $K=0$, which generalizes a theorem of Ehrenfeucht and Pelczyński and answers a question of M. Jarden.
\end{abstract}

A. Ehrenfeucht and A. Pelczyński answering a question of A. Mostowski have proved that a polynomial

$$
F(x)+G(y)+H(z),
$$

where $F, G, H$ are nonconstant polynomials over the complex field $C$ is irreducible over $C$. For the proof which extends to all fields of characteristic zero see [3] or [5]. In [4] (p. 53) the following generalization to fields of arbitrary characteristic has been proved. Let $K$ be a field and $F, G, H \in$ $K[x] \backslash K$. Then $F(x)+G(y)+H(z)$ is reducible over $K$ if and only if

$$
\begin{gathered}
F(x)-F(0)=L\left(F_{1}(x)\right), \quad G(y)-G(0)=L\left(G_{1}(y)\right), \\
H(z)-H(0)=L\left(H_{1}(z)\right),
\end{gathered}
$$

where $L \in K[t]$ is an additive polynomial, $F_{1} \in K[x], G_{1} \in K[y], H_{1} \in$ $K[z]$ and $L(t)+F(0)+G(0)+H(0)$ is reducible over $K$.

Let us adopt the following

Definition 1. A rational function is reducible over $K$ if the numerator in its reduced form is reducible over $K$.

Recently M. Jarden has asked whether with this definition polynomials in Ehrenfeucht and Pelczyński's theorem can be replaced by rational functions. We shall answer this question in the affirmative by proving a more general result concerning fields of arbitrary characteristic. This generalizes also the result quoted above. To formulate it we need some notation. 
Definition 2. For a rational function $f \in K(x)$ ord $f$ is the order of the pole of $f$ at infinity and $c(f)$ is the constant term in the Laurent expansion of $f$ at infinity, i.e. of $f$ viewed as an element of $K\left(\left(x^{-1}\right)\right)$.

TheOREM. Let $K$ be a field and let $n \geq 3, f_{l} \in K\left(x_{i}\right) \backslash K(i=$ $1,2, \ldots, n)$. The rational function $\sum_{i=1}^{n} f_{i}$ is reducible over $K$ if and only if at least one of the following three conditions is satisfied.

(i) There is an additive polynomial $L$ and rational functions $g_{i}(i=$ $1,2, \ldots, n)$ such that

(1) $L \in K[z], g_{i} \in K\left(x_{i}\right), f_{i}-c\left(f_{i}\right)=L\left(g_{\imath}\right)(i=1,2, \ldots, n)$ and

(2) $L+\sum_{i=1}^{n} c\left(f_{i}\right)$ is reducible over $K$.

(ii) char $K=2$ and there exist rational functions $g_{i} \in K\left(x_{i}\right) \quad(i=$ $1,2, \ldots, n)$ and elements $c, d, g_{0} \in K$ such that $\sqrt{d} \notin K$

$$
\begin{gathered}
f_{i}-c\left(f_{i}\right)=\frac{c}{g_{l}^{2}+d} \quad(i=1,2, \ldots, n), \\
\sum_{i=1}^{n} c\left(f_{i}\right)=0 \quad \text { or } \quad \frac{c}{g_{0}^{2}+d} .
\end{gathered}
$$

(iii) char $K=2$, ord $f_{i} \leq 0(i=1,2, \ldots, n)$, (1) and (2) hold with $K$ replaced by a quadratic inseparable extension $\tilde{K}$ of $K$ and $L+\sum_{i=1}^{n} c\left(f_{i}\right)$ is not a constant multiple of the square of a polynomial irreducible over $\tilde{K}$.

Corollary 1. If $K$ is a field of characteristic $0, n \geq 3$ and $f_{i} \in$ $K\left(x_{i}\right) \backslash K(i=1,2, \ldots, n)$ then $\sum_{l=1}^{n} f_{i}$ is irreducible over $K$.

COROLlARY 2. If $K$ is an algebraically closed field of characteristic $p, n \geq 3$ and $f_{i} \in K\left(x_{i}\right) \backslash K$, then $\sum_{i=1}^{n} f_{l}$ is reducible over $K$ if and only if $f_{i}(x)=h_{i}(x)^{p}+\operatorname{ch}_{i}(x)$, where $h_{i} \in K(x)(i=1,2, \ldots, n)$ and $c \in K$.

In the case of polynomials and $n=3$ the last corollary has been proved by Tverberg [6].

COROllary 3. If char $K \neq 2$ or $K$ is perfect (i) constitutes a necessary and sufficient condition of the reducibility of $\sum_{i=1}^{n} f_{i}$ over $K$.

Proofs of the corollaries are given towards the end of the paper. An example given at the end shows that for every field $K$ of characteristic two that is not perfect the case (iii) of the theorem actually occurs.

The proof of the theorem is rather long. It incorporates suggestions made by J. Browkin, R. Dvornicich and M. Jarden, which are gratefully 
acknowledged. For the case of $K$ of characteristic zero M. Fried has found a different proof based on Proposition 2 of his paper [0].

For the sake of brevity we introduce the following notations and notions.

Definition 3. For a polynomial $F \in K[x]|F|$ is the degree of $F, l(F)$ is the leading coefficient of $F$ and $F(u, v)=v^{|F|} F(u / v)$.

Definition 4. A rational function is strongly reducible over $K$ if the numerator in its reduced form is reducible over $K$ and not equal to a constant multiple of the square of a polynomial irreducible over $K$.

Definition 5. For a field $K, \hat{K}$ is its algebraic closure.

We begin with a generalization to rational functions of the Lemma to Theorem 11 (p. 50) of [4].

LEMMA 1. Let $f\left(x_{1}, \ldots, x_{k}\right)$ and $r\left(t, y_{1}, \ldots, y_{l}\right)$ be rational functions over $K$, the former non-constant the latter nonconstant and of non-negative order with respect to $t$ and to at least one $y_{j}$.

If the function

$$
r\left(f\left(x_{1}, \ldots, x_{k}\right) ; y_{1}, \ldots, y_{l}\right)
$$

is reducible (resp. strongly reducible) over $K$ then

$$
f=h(g), \quad h \in K(u), \quad g \in K\left(x_{1}, \ldots, x_{k}\right)
$$

and

$$
r\left(h(u) ; y_{1}, \ldots, y_{l}\right)
$$

is reducible (resp. strongly reducible) over $K$.

Proof. For the sake of brevity put $\left\langle x_{1}, \ldots, x_{k}\right\rangle=X,\left\langle y_{1}, \ldots, y_{l}\right\rangle=Y$, and let $r=R / R^{*}$, where $R, R^{*} \in K[t, Y],\left(R, R^{*}\right)=1$. Let $\rho, \rho^{*}$ be the degree with respect to $t$ of $R$ and $R^{*}$ respectively.

By the assumption, $\rho \geq \rho^{*}$. If

$$
f=\frac{F}{F^{*}}, \quad \text { where } F, F^{*} \in K[x],\left(F, F^{*}\right)=1
$$

we get

$$
r(f, Y)=\frac{F(f, Y) \cdot F^{* \rho}}{R^{*}(f, Y) \cdot F^{* \rho}}
$$


The conditions $\left(R, R^{*}\right)=\left(F, F^{*}\right)=1, \rho \geq \rho^{*}$ easily imply that the numerator and the denominator on the right hand side of (3) are coprime polynomials. Therefore, reducibility of $r(f, Y)$ over $K$ means that

$$
R(f, Y) \cdot F^{* \rho}=P(X, Y) \cdot Q(X, Y),
$$

where

$$
P, Q \in K[X, Y] \backslash K \text {. }
$$

Let

$$
\begin{array}{cc}
R(f, Y)=\sum_{i=0}^{m} A_{i}(f) M_{i}(Y), & A_{i} \in K[u], A_{0} \neq 0 \\
P(X, Y)=\sum_{i=0}^{p} B_{i} P_{i}(Y), & B_{i} \in K[X], B_{0} \neq 0 \\
Q(X, Y)=\sum_{j=0}^{q} C_{j} Q_{j}(Y), & C_{j} \in K[X], C_{0} \neq 0,
\end{array}
$$

where $M_{i}, P_{l}, Q_{l}$ are distinct products of powers of $y_{1}, \ldots, y_{l}$ ordered antilexicographically, so that $M_{0}, P_{0}, Q_{0}$ are first in order. By the assumption $M_{0} \notin K$.

Consider the greatest common factor $D(u)$ of the polynomials $A_{i}(u)$ $(0 \leq i \leq m)$. If $D(u) \neq 1$

$$
R(u, Y)=D(u) \cdot \sum_{i=0}^{m} \frac{A_{i}(u)}{D(u)} M_{i}(Y)
$$

is a factorization of $R(u, Y)$ and both assertions of the lemma hold with $h(u)=u, g=f$.

If $D(u)=1$ there exist polynomials $E_{i} \in K[u](0 \leq i \leq m)$ such that

$$
\sum_{i=0}^{m} A_{i}(u) E_{l}(u)=1 .
$$

Hence

$$
\sum_{i=0}^{m} A_{i}(f) E_{l}(f)=1
$$

and the greatest common divisor of $A_{t}(f) F^{* \rho}(0 \leq i \leq m)$ divides a power of $F^{*}$. However by the definition of $\rho, \rho=\left|a_{j}\right|$ for some $j \leq m$ and then $\left(A,(f) F^{* \rho}, F^{*}\right)=1$ since $\left(F, F^{*}\right)=1$. Therefore the polynomials $A_{l}(f) F^{* \rho}(0 \leq i \leq m)$ are relatively prime and by (4), (6) and (7) we have $\left(B_{0}, \ldots, B_{p}\right)=1$. Since $P_{0}$ is the first in the antilexicographic order among $P_{i}$ and by (5) $P \notin K$ it follows from (7) that $P_{0} \notin K$. Similarly $Q_{0} \notin K$. By 
Corollary 1 to Theorem 10 in [4] (p. 48) for all positive integers $i \leq p$ and $j \leq q$ there exist polynomials

$$
\Omega_{i}, \Omega_{j}^{*} \in K\left[t ; v_{1}, \ldots, v_{m}\right]
$$

with integral coefficients monic with respect to $t$ such that

$$
\begin{aligned}
& \Omega_{\imath}\left(\frac{B_{i}}{B_{0}}, \frac{A_{1}(f)}{A_{0}(f)}, \ldots, \frac{A_{m}(f)}{A_{0}(f)}\right)=0, \\
& \Omega_{j}^{*}\left(\frac{C_{j}}{C_{0}}, \frac{A_{1}(f)}{A_{0}(f)}, \ldots, \frac{A_{m}(f)}{A_{0}(f)}\right)=0 .
\end{aligned}
$$

It follows that the field

$$
K\left(\frac{B_{1}}{B_{0}}, \ldots, \frac{B_{p}}{B_{0}}, \frac{C_{1}}{C_{0}}, \ldots, \frac{C_{q}}{C_{0}}, f\right)
$$

is over $K$ of transcendence degree 1 and being contained in $K(X)$, in virtue of a theorem of Igusa [2] (for an elementary proof see [4], §3 and Appendix) it is generated over $K$ by a single function $g \in K(X) \backslash K$. Thus we get

$$
f=h(g), \quad \frac{B_{i}}{B_{0}}=b_{\imath}(g)(0 \leq i \leq p), \quad \frac{C_{J}}{C_{0}}=c_{J}(g)(0 \leq j \leq q),
$$

where $h, b_{i}, c_{j} \in K(t)(0 \leq i \leq p, 0 \leq j \leq q)$. Since $A_{0}(f) F^{* \rho}=B_{0} C_{0}$ the equation (4) takes the form

$$
R(h(g), Y)=A_{0}(h(g)) \cdot\left(\sum_{i=0}^{p} b_{i}(g) P_{i}(Y)\right) \cdot\left(\sum_{j=0}^{q} c_{j}(g) Q_{j}(Y)\right)
$$

and since $g \notin K$ we can pass to the equation

$$
R(h(u), Y)=A_{0}(h(u))\left(\sum_{i=0}^{p} b_{j}(u) P_{\imath}(Y)\right) \cdot\left(\sum_{j=0}^{q} c_{j}(u) Q_{J}(Y)\right) .
$$

Since both factors in brackets are of positive degree with respect to $Y$, the function $r(h(u), Y)$ is reducible over $K$. If $R(f, Y) F^{* \rho}$ is not a constant multiple of the square of an irreducible polynomial we can assume that $P Q^{-1} \notin K(X)$. Hence the ratio of the two brackets is not in $K(u)$ and $r(h(u), Y)$ is strongly reducible over $K$.

Lemma 2. If $\sum_{j=1}^{k} A_{j}(x) B_{j}(y)=0$, where $A_{j}, B_{j} \in K[t](1 \leq j \leq k)$ and $l$ is the dimension of the linear space spanned over $K$ by $A_{j}(1 \leq j \leq k)$, $m$ is the dimension of the linear space spanned over $K$ by $B_{j}(1 \leq j \leq k)$ then $l+m \leq k$. 
Proof. Assume that $A_{1}, \ldots, A_{l}$ are linearly independent over $K$ and that

$$
A_{\imath}=\sum_{j=1}^{l} a_{i j} A_{J}, \quad a_{i j} \in K(l<i \leq k, 1 \leq j \leq k) .
$$

We get

$$
\sum_{j=1}^{l} A_{j}(x)\left(B_{j}(y)+\sum_{i=l+1}^{k} a_{i j} B_{i}(y)\right)=0 .
$$

Since $A_{1}(x), \ldots, A_{l}(x)$ are linearly independent over $K$ they are linearly independent over $K(y)$ hence

$$
B_{j}(y)+\sum_{i=l+1}^{k} a_{i j} B_{l}(y)=0 \quad(1 \leq j \leq l)
$$

and $m \leq k-l$.

Lemma 3. Let $f \in K(z) \backslash K$, where $K$ is algebraically closed. There exists a homography (fractional linear transformation) $\chi \in K(x)$ such that ord $f(\chi)>0$.

Proof. Let $f=F / F^{*}$, where $F, F^{*} \in K[z]$ and $\left(F, F^{*}\right)=1$. If $\left|F^{*}\right|<$ $|F|$ we take $\chi(x)=x$. If $\left|F^{*}\right| \geq|F|$ we have $|F|^{*}>0$ hence there exists a $\xi \in K$ such that $F^{*}(\xi)=0$. Clearly $F(\xi) \neq 0$. We take

$$
\chi(X)=\frac{\xi x+1}{x}
$$

and get

$$
f(\chi(x))=\frac{F(\xi x+1, x) x^{|F|^{*}-|F|}}{F^{*}(\xi x+1, x)} .
$$

Now $F^{*}(\xi, 1)=0, F(\xi, 1) \neq 0$, hence the degree of the numerator of $f(\chi(x))$ is $|F|^{*}$, the degree of the denominator is smaller.

LEMMA 4. If $f_{l} \in K\left(x_{\imath}\right) \backslash K(i=1,2), K$ is algebraically closed and

(9) $f_{1}\left(x_{1}\right)+f_{2}\left(x_{2}\right)=h\left(g\left(x_{1}, x_{2}\right)\right), \quad h \in K(t), g \in K\left(x_{1}, x_{2}\right)$

then

$$
h=H(\chi), \quad g=\chi^{-1}\left(g_{1}\left(x_{1}\right)+g_{2}\left(x_{2}\right)\right),
$$

where $H \in K[z], g_{\imath} \in K\left(x_{i}\right)(i=1,2)$ and $\chi(t)=t$ or $1 /(t-\gamma)(\gamma \in K)$ depending on whether $h \in K[t]$ or not. 
Proof. Consider first the case, where $h$ is a polynomial. Suppose that (11) $f_{i}=F_{i} / F_{i}^{*}$, where $F_{i}, F_{i}^{*} \in K\left[x_{l}\right]$ and $\left(F_{i}, F_{i}^{*}\right)=1(i=1,2)$.

The assertion of the lemma is invariant with respect to rational transformation of $x_{i}(i=1,2)$, hence by Lemma 3 we may assume that $\left|F_{i}\right|>\left|F_{i}^{*}\right|$ $(i=1,2)$.

Let

$$
g=\frac{P}{P^{*}}, \quad P, P^{*} \in K\left[x_{1}, x_{2}\right],\left(P, P^{*}\right)=1 .
$$

Clearly the denominator of $h(g)$ is $P^{*|h|}$, on the other hand the denominator of $f_{1}+f_{2}$ is $F_{1}{ }^{*} F_{2}{ }^{*}$ since $\left(F_{1}^{*}\left(x_{1}\right), F_{2}^{*}\left(x_{2}\right)\right)=1$. Hence by (9)

$$
F_{1}^{*} F_{2}^{*} P^{*-|h|} \in K
$$

and

$$
P^{*}=Q_{1} Q_{2}, \text { where } Q_{\imath} \in K\left[x_{i}\right] .
$$

Now put in (9) $x_{1}=\xi_{1}$, where $\xi_{1} \in K$ is chosen so that $F_{1}^{*}\left(\xi_{1}\right) \neq 0$. We get

$$
f_{1}\left(\xi_{1}\right)+f_{2}\left(x_{2}\right)=h\left(g\left(\xi_{1}, x_{2}\right)\right)
$$

hence subtracting from $(9)$

$$
\begin{aligned}
& f_{1}\left(x_{1}\right)-f_{1}\left(\xi_{1}\right)=h\left(g\left(x_{1}, x_{2}\right)\right)-h\left(g\left(\xi_{1}, x_{2}\right)\right) \\
& \quad=\left[g\left(x_{1}, x_{2}\right)-g\left(\xi_{1}, x_{2}\right)\right] \cdot h_{1}\left(g\left(x_{1}, x_{2}\right), g\left(\xi_{1}, x_{2}\right)\right),
\end{aligned}
$$

where $h_{1}$ is the slope of $h$, hence a polynomial of degree $|h|-1$. We have by (12)

$$
\begin{aligned}
g\left(x_{1}, x_{2}\right)-g\left(\xi_{1}, x_{2}\right) & =\frac{P^{*}\left(\xi_{1}, x_{2}\right) P\left(x_{1}, x_{2}\right)-P\left(\xi_{1}, x_{2}\right) P^{*}\left(x_{1}, x_{2}\right)}{P^{*}\left(\xi_{1}, x_{2}\right) P^{*}\left(x_{1}, x_{2}\right)} \\
& =\frac{P\left(x_{1}, x_{2}\right) Q_{1}\left(\xi_{1}\right)-P\left(\xi_{1}, x_{2}\right) Q_{1}\left(x_{1}\right)}{Q_{1}\left(\xi_{1}\right) Q_{1}\left(x_{1}\right) Q_{2}\left(x_{2}\right)}
\end{aligned}
$$

and the denominator of $h_{1}\left(g\left(x_{1}, x_{2}\right), g\left(\xi_{1}, x_{2}\right)\right)$ divides $\left(Q_{1} Q_{2}\right)^{|h|-1}$. It follows from (14) that

$$
P\left(x_{1}, x_{2}\right) Q_{1}\left(\xi_{1}\right)-P\left(\xi_{1}, x_{2}\right) Q_{1}\left(x_{1}\right) \mid\left(F_{1}\left(x_{1}\right) F_{1}^{*}\left(\xi_{1}\right)-F_{1}^{*}\left(x_{1}\right) F_{1}\left(\xi_{1}\right)\right) Q_{2}^{|h|} \text {. }
$$

Thus

$$
P\left(x_{1}, x_{2}\right) Q_{1}\left(\xi_{1}\right)-P\left(\xi_{1}, x_{2}\right) Q_{1}\left(x_{1}\right)=A_{1} B_{2},
$$

where $A_{1} \in K\left[x_{1}\right], B_{2} \in K\left[x_{2}\right]$ and

$$
B_{2} \mid Q_{2}^{|h|} \text {. }
$$


Similarly choosing $\xi_{2}$ so that $F_{2}{ }^{*}\left(\xi_{2}\right) \neq 0$ we find

$$
P\left(x_{1}, x_{2}\right) Q_{2}\left(\xi_{2}\right)-P\left(x_{1}, \xi_{2}\right) Q_{2}\left(x_{2}\right)=A_{2} B_{1},
$$

where $A_{2} \in K\left[x_{2}\right], B_{1} \in K\left[x_{1}\right]$ and

$$
B_{1} \mid Q_{1}^{|h|} \text {. }
$$

Eliminating $P\left(x_{1}, x_{2}\right)$ from the formulae $\left(15_{1}\right)$ and $\left(15_{2}\right)$ we get

$$
\begin{aligned}
Q_{2}\left(\xi_{2}\right) P\left(\xi_{1}, x_{2}\right) Q_{1}\left(x_{1}\right)+Q_{2}\left(\xi_{2}\right) A_{1}\left(x_{1}\right) B_{2}\left(x_{2}\right) \\
=Q_{1}\left(\xi_{1}\right) P\left(x_{1}, \xi_{2}\right) Q_{2}\left(x_{2}\right)+Q_{1}\left(\xi_{1}\right) A_{2}\left(x_{2}\right) B_{1}\left(x_{1}\right) .
\end{aligned}
$$

Let $l_{i}$ be the dimension of the linear space spanned over $K$ by $A_{i}, B_{i}, Q_{i}$, $\left.P\left(x_{1}, x_{2}\right)\right|_{x_{3-1}=\xi_{3-l}}(i=1,2)$. By Lemma 2 we have

$$
l_{1}+l_{2} \leq 4 \text {. }
$$

If for $i=1$ or $i=2$ we had $l_{i}=1$ it would follow that $A_{i} / Q_{i} \in K$, hence by $\left(15_{i}\right) Q_{i} \mid P$ and by (12) and (13) $Q_{i} \in K, A_{\imath} \in K$. Thus by $\left(15_{l}\right)$ $P \in K\left[x_{3-i}\right]$, by (13) $P^{*} \in K\left[x_{3-i}\right]$ and by (12) $g \in K\left(x_{3-i}\right)$ contrary to (9) and $f_{i} \notin K$. Therefore

$$
l_{1}=l_{2}=2 .
$$

If, for an $i \leq 2, B_{3-i}, Q_{3-i}$ are linearly dependent then $B_{3-i}=c Q_{3-i}$, $c \in K$, and $\left(15_{i}\right)$ gives

$$
\frac{P\left(x_{1}, x_{2}\right)}{P^{*}\left(x_{1}, x_{2}\right)}=\frac{\left.P\left(x_{1}, x_{2}\right)\right|_{x_{i}=\xi_{l}}}{Q_{i}\left(\xi_{i}\right) Q_{3-i}\left(x_{3-i}\right)}+\frac{c A_{i}\left(x_{i}\right)}{Q_{i}\left(\xi_{l}\right) Q_{i}\left(x_{i}\right)}
$$

thus (10) holds with $H=h, \chi(t)=t$ and $g_{j}\left(x_{j}\right)$ equal to the first or the second term on the right hand side of (18) depending on whether $j=3-i$ or $j=i$.

If, for an $i \leq 2, B_{3-i}$ and $Q_{3-i}$ are linearly independent then since $l_{3-1}=2,\left.P\left(x_{1}, x_{2}\right)\right|_{x_{t}=\xi_{t}}$ must be their linear combination over $K$ hence $\left(B_{3-i}, Q_{3-i}\right)$ divides $\left.P\left(x_{1}, x_{2}\right)\right|_{x_{1}=\xi_{1}}$ and by $\left(15_{i}\right)\left(B_{3-i}, Q_{3-i}\right)$ divides $P\left(x_{1}, x_{2}\right)$. Since $\left(Q_{3-i}, P\right)=1$ by (12) and (13) it follows that $\left(B_{3-i}, Q_{3-i}\right)=1$ and, by $\left(16_{i}\right), B_{3-i} \in K \backslash\{0\}, Q_{3-i} \notin K$.

Thus for $h$ being a polynomial it remains to consider the case

$$
B_{1}, B_{2} \in K \backslash\{0\}, \quad Q_{1}, Q_{2} \notin K .
$$

In this case the condition $l_{1}=l_{2}=2$ gives for $i=1$ and 2

$$
\begin{gathered}
A_{i}\left(x_{i}\right)=a_{\imath} Q_{i}\left(x_{i}\right)+b_{i} B_{i} \\
\left.P\left(x_{1}, x_{2}\right)\right|_{x_{3-l}=\xi_{3-l}}=c_{i} Q_{i}\left(x_{i}\right)+d_{\imath} B_{i},
\end{gathered}
$$


where $a_{i}, b_{i}, c_{i}, d_{i} \in K(i=1,2)$. Substituting this into (17) and compairing the coefficients of $Q_{i}\left(x_{l}\right)$ on both sides we get

$$
a_{i}+d_{3-\imath}=0 \quad(i=1,2) .
$$

It follows now from $\left(15_{1}\right)$ that

$$
P\left(x_{1}, x_{2}\right) Q_{1}\left(\xi_{1}\right)=c_{2} Q_{1}\left(x_{1}\right) Q_{2}\left(x_{2}\right)+b_{1} B_{1} B_{2},
$$

hence by (9), (12) and (13)

$$
f\left(x_{1}\right)+f_{2}\left(x_{2}\right)=h\left(\frac{c_{2}}{Q_{1}\left(\xi_{1}\right)}+\frac{b_{1} B_{1} B_{2}}{Q_{1}\left(\xi_{1}\right) Q_{1}\left(x_{1}\right) Q_{2}\left(x_{2}\right)}\right) .
$$

The order of the left hand side with respect to $x_{1}$ is positive, the order of the right-hand side at most 0 , thus we get a contradiction.

Consider now the case, where $h \notin K[t]$,

$$
h=\frac{S(t)}{S^{*}(t)}, \quad\left(S, S^{*}\right)=1 .
$$

If $|S| \leq\left|S^{*}\right|$ and $S^{*}=c(t-\gamma)^{\left|S^{*}\right|}$ then if we make the substitution $t=\gamma+z^{-1}$ we get

$$
h(t)=\frac{S_{0}(z)}{S_{0}^{*}(z)}=h_{0}(z),
$$

where $S_{0}=z^{\left|S^{*}\right|} S\left(\gamma+z^{-1}\right), S_{0}^{*}=z^{\left|S^{*}\right|} S^{*}\left(\gamma+z^{-1}\right) \in K$. Since $h_{0} \in K[z]$ and

$$
f_{1}\left(x_{1}\right)+f_{2}\left(x_{2}\right)=h_{0}\left(\frac{1}{g\left(x_{1}, x_{2}\right)-\gamma}\right)
$$

we get by the already proved case of the lemma

$$
\frac{1}{g\left(x_{1}, x_{2}\right)-\gamma}=g_{1}\left(x_{1}\right)+g_{2}\left(x_{2}\right)
$$

thus (10) holds with $H=h_{0}, \chi=1 /(t-\gamma)$.

If $|S|>\left|S^{*}\right|$ or $S^{*}$ has at least two distinct zeros then the denominator of $h(g)$ has by (12) a factor of the form $\left(\beta_{1} P+\beta_{1}^{*} P^{*}\right)\left(\beta_{2} P+\beta_{2}^{*} P^{*}\right)$, where $\beta_{l}, \beta_{i}^{*} \in K$ and $\beta_{1} \beta_{2}^{*}-\beta_{1}^{*} \beta_{2} \neq 0$. Since the denominator of $f_{1}+f_{2}$ is $F_{1}{ }^{*} F_{2}{ }^{*}$ we get by (9) for $i=1,2$

$$
\beta_{l} P+\beta_{l}^{*} P^{*}=Q_{i} Q_{3-i}^{*}, \quad \text { where } Q_{i}, Q_{i}^{*} \in K\left[x_{l}\right] \backslash\{0\} .
$$

It follows that

$$
P=\alpha_{1} Q_{1} Q_{2}^{*}+\alpha_{2} Q_{1}^{*} Q_{2}, \quad P^{*}=\alpha_{1}^{*} Q_{1}^{*} Q_{2}+\alpha_{2}^{*} Q_{1} Q_{2}^{*},
$$


where $\alpha_{i}, \quad \alpha_{i}^{*} \in K(i=1,2), \quad \alpha_{1} \alpha_{1}^{*}-\alpha_{2} \alpha_{2}^{*} \neq 0$. Now comparing the numerators of the two sides of (9) we get by (12) and (19)

$$
\begin{aligned}
F\left(x_{1}, x_{2}\right) & =F_{1}\left(x_{1}\right) F_{2}^{*}\left(x_{2}\right)+F_{1}^{*}\left(x_{1}\right) F_{2}\left(x_{2}\right) \\
& =\beta S\left(P, P^{*}\right) P^{* \max \left(0,\left|S^{*}\right|-|S|\right)} .
\end{aligned}
$$

Since $\left(F_{1}, F_{1}^{*}\right)=1, F_{1} F_{1}^{*} \notin K$ we cannot have $F \in K$. Let $G$ be a factor of $F$ irreducible over $K$. Now we shall use an argument taken from [1].

Consider the field $M$ of algebraic functions on the curve $G\left(\bar{x}_{1}, \bar{x}_{2}\right)=0$. Let $L$ be the subfield of $M$ equal to $K\left(\bar{x}_{1}\right) \cap K\left(\bar{x}_{2}\right)$.

In virtue of Lüroth's theorem $L / K$ has a single generator $w=\rho_{i}\left(\bar{x}_{i}\right)$, where $\rho_{i} \in K\left(\bar{x}_{i}\right)(i=1,2)$.

If $G\left(\bar{x}_{1}, \bar{x}_{2}\right)=0$ then $F\left(\bar{x}_{1}, \bar{x}_{2}\right)=0$ and since $G+F_{1}^{*} F_{2}^{*}$ by (11) and (21)

$$
f_{1}\left(\bar{x}_{1}\right)=-f_{2}\left(\bar{x}_{2}\right) \in L
$$

hence

$$
(-1)^{l-1} f_{l}\left(\bar{x}_{i}\right)=\tau(w), \quad \tau \in K(w) .
$$

It follows in particular that $w \notin K, \rho_{\imath} \notin K(i=1,2)$.

On the other hand, by (21)

$$
S\left(P\left(\bar{x}_{1}, \bar{x}_{2}\right), P^{*}\left(\bar{x}_{1}, \bar{x}_{2}\right)\right) P^{*}\left(\bar{x}_{1}, \bar{x}_{2}\right)^{\max \left(0,\left|S^{*}\right|-|S|\right)}=0 .
$$

The left-hand side is the product of linear forms in $P\left(\bar{x}_{1}, \bar{x}_{2}\right), P^{*}\left(\bar{x}_{1}, \bar{x}_{2}\right)$, hence for suitable $\delta, \delta^{*} \in K$ not both 0 we have

$$
\delta P\left(\bar{x}_{1}, \bar{x}_{2}\right)+\delta^{*} P^{*}\left(\bar{x}_{1}, \bar{x}_{2}\right)=0 .
$$

Hence by (20)

$$
\gamma_{1} Q_{1}\left(\bar{x}_{1}\right) Q_{2}^{*}\left(\bar{x}_{2}\right)-\gamma_{2} Q_{1}^{*}\left(\bar{x}_{1}\right) Q_{2}\left(\bar{x}_{2}\right)=0
$$

where

$$
\gamma_{1}=\delta \alpha_{1}+\delta^{*} \alpha_{2}^{*}, \quad \gamma_{2}=-\delta \alpha_{2}-\delta^{*} \alpha_{1}^{*} .
$$

If $\gamma_{1}=0$ or $\gamma_{2}=0$ then $\gamma_{1}=\gamma_{2}=0$ since $\Pi_{i=1}^{2} Q_{i} Q_{i}^{*} \neq 0$. Since $\alpha_{1} \alpha_{1}^{*}-$ $\alpha_{2} \alpha_{2}^{*} \neq 0$ this gives $\delta=\delta^{*}=0$, a contradiction. Thus

$$
\gamma_{i} \in K \backslash\{0\} \quad(i=1,2) \text {. }
$$

Moreover

$$
\gamma_{1} \frac{Q_{1}\left(\bar{x}_{1}\right)}{Q_{1}^{*}\left(\bar{x}_{1}\right)}=\gamma_{2} \frac{Q_{2}\left(\bar{x}_{2}\right)}{Q_{2}^{*}\left(\bar{x}_{2}\right)}
$$


and thus

$$
\gamma_{i} \frac{Q_{i}\left(\bar{x}_{i}\right)}{Q_{i}^{*}\left(\bar{x}_{1}\right)}=\tau_{0}(w), \quad \tau_{0} \in K(w), i=1,2
$$

Substituting $w=\rho_{i}\left(\bar{x}_{i}\right)$ we get from (22) and (23)

$$
\begin{array}{cc}
(-1)^{i-1} f_{i}\left(\bar{x}_{i}\right)=\tau\left(\rho_{i}\left(\bar{x}_{i}\right)\right) & (i=1,2), \\
\gamma_{i} \frac{Q_{i}\left(\bar{x}_{i}\right)}{Q_{i}^{*}\left(\bar{x}_{i}\right)}=\tau_{0}\left(\rho_{i}\left(\bar{x}_{i}\right)\right) & (i=1,2) .
\end{array}
$$

The obtained identities are clearly independent of the equation $G\left(\bar{x}_{1}, \bar{x}_{2}\right)$ $=0$ thus we can replace in them $\bar{x}_{i}$ by $x_{i}$. Substituting into (9) by means of (12) and (20) we get

$$
\tau\left(\rho_{1}\left(x_{1}\right)\right)-\tau\left(\rho_{2}\left(x_{2}\right)\right)=h\left(\frac{\alpha_{1} \gamma_{1}^{-1} \tau_{0}\left(\rho_{1}\left(x_{1}\right)\right)+\alpha_{2} \gamma_{2}^{-1} \tau_{0}\left(\rho_{2}\left(x_{2}\right)\right)}{\alpha_{2}^{*} \gamma_{1}^{-1} \tau_{0}\left(\rho_{1}\left(x_{1}\right)\right)+\alpha_{1}^{*} \gamma_{2}^{-1} \tau_{0}\left(\rho_{2}\left(x_{2}\right)\right)}\right) .
$$

Since $\rho_{1}\left(x_{1}\right), \rho_{2}\left(x_{2}\right)$ are algebraically independent it follows that for independent variables $u_{1}, u_{2}$ we have the identity

$$
\begin{aligned}
\tau\left(u_{1}\right)-\tau\left(u_{2}\right)= & h_{0}\left(\frac{\tau_{0}\left(u_{1}\right)}{\tau_{0}\left(u_{2}\right)}\right) \\
& \text { where } h_{0}(z)=h\left(\frac{\alpha_{1} \gamma_{1}^{-1} z+\alpha_{2} \gamma_{2}^{-1}}{\alpha_{2}^{*} \gamma_{1}^{-1} z+\alpha_{1}^{*} \gamma_{2}^{-1}}\right) .
\end{aligned}
$$

Replacing if necessary $u_{i}$ by $\chi\left(u_{l}\right)(i=1,2)$, where $\chi$ is a suitable homography we may assume by Lemma 3 that

$$
\begin{aligned}
& \tau_{0}\left(u_{\imath}\right)=\frac{T_{0}\left(u_{i}\right)}{T_{0}^{*}\left(u_{i}\right)} \quad(i=1,2), \\
& \quad \text { where }\left|T_{0}\right|>\left|T_{0}^{*}\right| \text { and }\left(T_{0}, T_{0}^{*}\right)=1 .
\end{aligned}
$$

Thus

$$
\frac{\tau_{0}\left(u_{1}\right)}{\tau_{0}\left(u_{2}\right)}=\frac{T_{0}\left(u_{1}\right) T_{0}^{*}\left(u_{2}\right)}{T_{0}^{*}\left(u_{1}\right) T_{0}\left(u_{2}\right)}
$$

Let moreover

$$
\tau(u)=\frac{T(u)}{T^{*}(u)}, \quad\left(T, T^{*}\right)=1
$$

If $h_{0}$ had a pole $p \neq 0, \infty$ then in virtue of (24) and (26) $\tau\left(u_{1}\right)-\tau\left(u_{2}\right)$ would have in the denominator the factor $T_{0}\left(u_{1}\right) T_{0}^{*}\left(u_{2}\right)-p T_{0}^{*}\left(u_{1}\right) T_{0}\left(u_{2}\right)$. 
Since the denominator of $\tau\left(u_{1}\right)-\tau\left(u_{2}\right)$ is $T^{*}\left(u_{1}\right) T^{*}\left(u_{2}\right)$ we would get

$$
T_{0}\left(u_{1}\right) T_{0}^{*}\left(u_{2}\right)-p T_{0}^{*}\left(u_{1}\right) T_{0}\left(u_{2}\right)=C_{1}\left(u_{1}\right) C_{2}\left(u_{2}\right), \quad C_{i} \in K\left[u_{i}\right]
$$

and in virtue of Lemma 2 for $i=1$ or $i=2 T_{0}\left(u_{l}\right), T_{0}^{*}\left(u_{i}\right)$ and $C_{i}\left(u_{l}\right)$ would span over $K$ a linear space of dimension 1, which contradicts (25).

Therefore $h_{0}$ has no pole except at 0 and $\infty$ and thus

$$
h_{0}(z)=\frac{R(z)}{z^{r}}, \quad \text { where } R \in K[z],
$$

Substituting this in (24) and taking into account (26) and (27) we get for an $\alpha \in K \backslash\{0\}$

$$
\begin{aligned}
& T\left(u_{1}\right) T^{*}\left(u_{2}\right)-T^{*}\left(u_{1}\right) T\left(u_{2}\right) \\
& \quad=\alpha R\left(T_{0}\left(u_{1}\right) T_{0}^{*}\left(u_{2}\right), T_{0}^{*}\left(u_{1}\right) T_{0}\left(u_{2}\right)\right)\left(T_{0}^{*}\left(u_{1}\right) T_{0}\left(u_{2}\right)\right)^{\max (0, r-|R|)} . \\
& T^{*}\left(u_{1}\right) T^{*}\left(u_{2}\right)=\alpha\left(T_{0}\left(u_{1}\right) T_{0}^{*}\left(u_{2}\right)\right)^{r}\left(T_{0}^{*}\left(u_{1}\right) T_{0}\left(u_{2}\right)\right)^{\max (0,|R|-r)} .
\end{aligned}
$$

The second equation gives for suitable $\varepsilon, \varepsilon^{*} \in \mathrm{K} \backslash\{0\}$

$$
\varepsilon T_{0}^{r} T_{0}^{* \max (0,|R|-r)}=T^{*}=\varepsilon^{*} T_{0}^{* r} T_{0}^{\max (0,|R|-r)}
$$

hence by (25)

$$
r=\max \{0,|R|-r\} ; \quad|R|=2 r, \quad\left|T^{*}\right|=r\left(\left|T_{0}\right|+\left|T_{0}^{*}\right|\right) .
$$

Consider now the equation (28). The degree of the right-hand side with respect to $u_{1}$ is in virtue of (25) equal to

$$
|R|\left|T_{0}\right|=2 r\left|T_{0}\right|>r\left(\left|T_{0}\right|+\left|T_{0}^{*}\right|\right)=\left|T^{*}\right|,
$$

the degree of the left-hand side does not exceed $\max \left(|T|,\left|T^{*}\right|\right)$ and is equal to it, if $|T| \neq\left|T^{*}\right|$, hence

$$
|T|=2 r\left|T_{0}\right| \text {. }
$$

Now the total degree of the left-hand side of (28) is by (30) and (31)

$$
|T|+\left|T^{*}\right|=r\left(3\left|T_{0}\right|+\left|T_{0}^{*}\right|\right),
$$

the total degree of the right-hand side is at most

$$
|R|\left(\left|T_{0}\right|+\left|T_{0}^{*}\right|\right)=r\left(2\left|T_{0}\right|+2\left|T_{0}^{*}\right|\right)<r\left(3\left|T_{0}\right|+\left|T_{0}^{*}\right|\right) .
$$

The obtained contradiction completes the proof.

Lemma 5. If $H \in K[t], f_{i} \in K\left(x_{i}\right), g_{i} \in K\left(x_{i}\right) \backslash K(i=1,2)$ and

$$
f_{1}\left(x_{1}\right)+f_{2}\left(x_{2}\right)=H\left(g_{1}\left(x_{1}\right)+g_{2}\left(x_{2}\right)\right)
$$


then

$$
H-H(0) \text { is an additive polynomial }
$$

and

$$
\begin{gathered}
f_{i}-c\left(f_{i}\right)=H\left(g_{i}-c\left(g_{i}\right)\right)-H(0) \quad(i=1,2), \\
c\left(f_{1}\right)+c\left(f_{2}\right)=H\left(c\left(g_{1}\right)+c\left(g_{2}\right)\right) .
\end{gathered}
$$

Proof. (Due to J. Browkin*). We prove the assertion (33) by induction with respect to $|H|$. If $|H| \leq 1$ the assertion is obvious. Assume that it true for all $H$ of degree less than $d>1$ and consider an $H$ with $|H|=d$.

Let $m$ be the greatest exponent such that $g_{2} \in K\left(x_{2}^{m}\right)$. We have

$$
g_{2}=g_{3}\left(x_{2}^{m}\right), \quad \text { where } g_{3} \in K\left(x_{3}\right) \backslash K
$$

and by (32)

$$
f_{2}=f_{3}\left(x_{2}^{m}\right), \quad f_{3} \in K\left(x_{3}\right)
$$

Moreover

$$
g_{3}^{\prime} \neq 0
$$

since otherwise char $K=p, g_{3} \in K\left(x_{3}^{p}\right)$ and $g_{2} \in K\left(x_{2}^{p m}\right)$ contrary to the choice of $m$. It follows from (32), (36) and (37) that

$$
f_{1}+f_{3}=H\left(g_{1}+g_{3}\right) \text {. }
$$

Differentiating with respect to $x_{3}$ we get

$$
f_{3}^{\prime}=H^{\prime}\left(g_{1}+g_{3}\right) g_{3}^{\prime} \text {. }
$$

In virtue of (37) and (38) we get $H^{\prime}\left(g_{1}+g_{3}\right) \in K\left(x_{3}\right)$ and since $g_{1} \notin$ $K\left(x_{3}\right)$ it follows that $H^{\prime} \in K$. Thus char $K=p$ and

$$
H=H^{\prime}(0) t+H_{0}\left(t^{p}\right), \quad H_{0} \in K(u) .
$$

By (39)

$$
f_{1}-H^{\prime}(0) g_{1}+f_{3}-H^{\prime}(0) g_{3}=H_{0}\left(g_{1}^{p}+g_{3}^{p}\right) \text {. }
$$

However by (40) $\left|H_{0}\right|=d / p<d$, hence by the inductive assumption $H_{0}-H_{0}(0)$ is an additive polynomial and by $(40) H-H(0)$ is also one.

In order to prove the last part of the lemma we observe that by the additivity of $H(t)-H(0)$ we have

$$
\begin{aligned}
f_{1}+f_{2} & =H\left(g_{1}-c\left(g_{1}\right)+g_{2}-c\left(g_{2}\right)\right)+H\left(c\left(g_{1}\right)+c\left(g_{2}\right)\right)-H(0) \\
& =\sum_{i=1}^{2}\left(H\left(g_{i}-c\left(g_{i}\right)\right)-H(0)\right)+H\left(c\left(g_{1}\right)+c\left(g_{2}\right)\right) .
\end{aligned}
$$

\footnotetext{
* The author's original proof was valid only for $K$ algebraically closed.
} 
Now we expand both sides into Laurent series in powers of $x_{1}$ and $x_{2}$ and since

$$
c\left(H\left(g_{i}-c\left(g_{i}\right)\right)-H(0)\right)=0
$$

we infer (34) and (35).

LeMMA 6. If $K$ is a field

$$
\begin{gathered}
g_{\imath} \in \hat{K}\left(x_{i}\right) \backslash \hat{K} \quad(i=1,2), \quad \gamma \in \hat{K} \\
\gamma+\frac{1}{g_{1}+g_{2}} \in K\left(x_{1}, x_{2}\right)
\end{gathered}
$$

then either

$$
\gamma \in K, \quad g_{i}-c\left(g_{l}\right) \in K\left(x_{i}\right)(i=1,2), \quad c\left(g_{1}\right)+c\left(g_{2}\right) \in K
$$

or

$$
\begin{gathered}
\operatorname{char} K=2, \quad \gamma \notin K, \quad \gamma^{2} \in K, \\
g_{i}-c\left(g_{i}\right)=\frac{1}{h_{i}+\gamma}, \quad h_{i} \in K\left(x_{i}\right)(i=1,2) \\
c\left(g_{1}\right)+c\left(g_{2}\right)=0 \text { or } \frac{1}{h_{0}+\gamma}, \quad h_{0} \in K .
\end{gathered}
$$

Proof. If $\gamma \in K$ then clearly

$$
g_{1}+g_{2} \in K\left(x_{1}, x_{2}\right)
$$

hence $g_{i}-c\left(g_{i}\right) \in K\left(x_{1}\right)(i=1,2), c\left(g_{1}\right)+c\left(g_{2}\right) \in K$. Therefore we assume that

$$
\gamma \notin K
$$

Let

$$
g_{i}=\frac{G_{i}}{G_{i}^{*}}, \quad G_{i}, G_{i} \in K\left[x_{i}\right],\left(G_{i}, G_{i}^{*}\right)=1(i=1,2) .
$$

We assume without loss of generality that $l\left(G_{i}^{*}\right)=1(i=1,2)$.

If for an $i \leq 2$ we had $\left|G_{l}\right|>\left|G_{i}^{*}\right|$ then the leading coefficient with respect to $x_{i}$ in the numerator of $\gamma+1 /\left(g_{1}+g_{2}\right)$ would be $\gamma l\left(G_{i}\right) G_{3-i}^{*}$, in the denominator $l\left(G_{l}\right) G_{3-i}^{*}$, hence $\gamma \in K$ contrary to (42). Therefore $\left|G_{i}\right| \leq\left|G_{i}^{*}\right|(i=1,2)$ and we have for $i=1,2$

$$
G_{i}=\alpha_{i} G_{i}^{*}+H_{i}, \quad \alpha_{i}=c\left(g_{i}\right), H_{i} \in \hat{K}\left[x_{i}\right] \text { and }-\infty<\left|H_{\imath}\right|<\left|G_{i}^{*}\right| .
$$

Thus we get

$$
\gamma+\frac{1}{g_{1}+g_{2}}=\frac{\gamma\left(H_{1} G_{2}^{*}+G_{1}^{*} H_{2}\right)+\left(\gamma \alpha_{1}+\gamma \alpha_{2}+1\right) G_{1}^{*} G_{2}^{*}}{H_{1} G_{2}^{*}+G_{1}^{*} H_{2}+\left(\alpha_{1}+\alpha_{2}\right) G_{1}^{*} G_{2}^{*}}
$$


If $\alpha_{1}+\alpha_{2} \neq 0$ the leading coefficient with respect to $x_{1}$ in the denominator is $\left(\alpha_{1}+\alpha_{2}\right) G_{2}^{*}+H_{2}$ in the numerator is $\left(\gamma \alpha_{1}+\gamma \alpha_{2}+1\right) G_{2}^{*}+\gamma H_{2}$ unless $\gamma \alpha_{1}+\gamma \alpha_{2}+1=0$, hence

$$
\begin{aligned}
\frac{l\left(\left(\gamma \alpha_{1}+\gamma \alpha_{2}+1\right) G_{2}^{*}+\gamma H_{2}\right)}{l\left(\left(\alpha_{1}+\alpha_{2}\right) G_{2}^{*}+H_{2}\right)} & =\frac{\gamma \alpha_{1}+\gamma \alpha_{2}+1}{\alpha_{1}+\alpha_{2}} \\
& =\gamma+\frac{1}{\alpha_{1}+\alpha_{2}} \in K^{*}
\end{aligned}
$$

We have in this case

$$
\begin{gathered}
\frac{\gamma}{\alpha_{1}+\alpha_{2}}\left(H_{1} G_{2}^{*}+G_{1}^{*} H_{2}\right)+\frac{\gamma \alpha_{1}+\gamma \alpha_{2}+1}{\alpha_{1}+\alpha_{2}} G_{1}^{*} G_{2}^{*} \in K\left[x_{1}, x_{2}\right], \\
\frac{1}{\alpha_{1}+\alpha_{2}}\left(H_{1} G_{2}^{*}+G_{1}^{*} H_{2}\right)+G_{1}^{*} G_{2}^{*} \in K\left[x_{1}, x_{2}\right] .
\end{gathered}
$$

Multiplying the second relation by $\left(\gamma \alpha_{1}+\gamma \alpha_{2}+1\right) /\left(\alpha_{2}+\alpha_{2}\right)$ and subtracting from the first we get

$$
\frac{1}{\left(\alpha_{1}+\alpha_{2}\right)^{2}}\left(H_{1} G_{2}^{*}+G_{1}^{*} H_{2}\right) \in K\left[x_{1}, x_{2}\right] .
$$

The leading coefficient with respect to $x_{t}$ of the polynomial on the left-hand side is

$$
\frac{1}{\left(\alpha_{1}+\alpha_{2}\right)^{2}} H_{3-i}\left(x_{3-i}\right) \text {. }
$$

Hence

$$
\frac{1}{\left(\alpha_{1}+\alpha_{2}\right)^{2}} H_{i} \in K\left[x_{i}\right] \quad(i=1,2) .
$$

Let $1=\omega_{1}, \omega_{2}, \ldots, \omega_{k}$ be a basis of the linear space spanned over $K$ by the coefficients of $G_{1}^{*}, G_{2}^{*}$ and let

$$
G_{i}^{*}=\sum_{j=1}^{k} G_{i j}^{*} \omega_{\jmath}, \quad G_{i j}^{*} \in K\left[x_{i}\right](i=1,2) .
$$

The condition (48) gives

$$
\sum_{j=1}^{k}\left(\frac{1}{\left(\alpha_{1}+\alpha_{2}\right)^{2}} H_{1} G_{2 j}^{*}+\frac{1}{\left(\alpha_{1}+\alpha_{2}\right)^{2}} G_{1 j}^{*} H_{2}\right) \omega_{j} \in K\left[x_{1}, x_{2}\right]
$$

hence by (49)

$$
H_{1} G_{2,}^{*}+G_{1 j}^{*} H_{2}=0 \quad(1<j \leq k) .
$$

* In the original manuscript there was an error detected and corrected by R. Dvornicich. 
This implies by Lemma 2

$$
G_{1 j}^{*}=\beta_{j} H_{1}, \quad G_{2 j}^{*}=-\beta, H_{2}, \quad \beta_{j} \in \hat{K}(1<j \leq k),
$$

by (50) and (44)

$$
G_{1}^{*}=G_{11}^{*}+\alpha H_{1}, \quad G_{2}^{*}=G_{21}^{*}-\alpha H_{2}, \quad \alpha \in \hat{K}
$$

and by (44)

$$
\left|H_{i}\right|<\left|G_{i 1}^{*}\right| \quad(i=1,2) .
$$

The condition (47) gives

$$
\begin{aligned}
& \frac{1}{\alpha_{1}+\alpha_{2}}\left(H_{1} G_{21}^{*}+G_{11}^{*} H_{2}\right)+G_{11}^{*} G_{21}^{*} \\
& \quad+\alpha\left(H_{1} G_{21}^{*}-G_{11}^{*} H_{2}\right)-\alpha^{2} H_{1} H_{2} \in K\left[x_{1}, x_{2}\right]
\end{aligned}
$$

hence

$$
\left(\frac{1}{\alpha_{1}+\alpha_{2}}+\alpha\right) H_{1} G_{21}^{*}+\left(\frac{1}{\alpha_{1}+\alpha_{2}}-\alpha\right) G_{11}^{*} H_{2}-\alpha^{2} H_{1} H_{2} \in K\left[x_{1}, x_{2}\right] .
$$

Considering the leading coefficients with respect to $x_{1}$ and $x_{2}$ we find by (52)

$$
\left(\frac{1}{\alpha_{1}+\alpha_{2}}+\alpha\right) H_{1} \in K\left[x_{1}\right], \quad\left(\frac{1}{\alpha_{1}+\alpha_{2}}-\alpha\right) H_{2} \in K\left[x_{2}\right]
$$

hence also

$$
\alpha^{2} H_{1} H_{2} \in K\left[x_{1}, x_{2}\right]
$$

A comparison with (49) gives

$$
\alpha_{1}+\alpha_{2}+\alpha\left(\alpha_{1}+\alpha_{2}\right)^{2} \in K, \quad \alpha_{1}+\alpha_{2}-\alpha\left(\alpha_{1}+\alpha_{2}\right)^{2} \in K
$$

hence

$$
2\left(\alpha_{1}+\alpha_{2}\right) \in K
$$

By (42), (46) and (55) char $K=2$. On the other hand, by (49) and (55) $\alpha^{2}\left(\alpha_{1}+\alpha_{2}\right)^{4} \in K$ and then by (54) $\left(\alpha_{1}+\alpha_{2}\right)^{2} \in K$, by (46) $\gamma^{2} \in K$ and (41a) holds. Moreover by (54) $1 /\left(\alpha_{1}+\alpha_{2}\right)+\alpha \in K$, hence by (46) $\gamma+\alpha$ $\in K$ and (41b) holds in virtue of (43), (44), (49)-(51) with $h_{i}=G_{i 1}^{*} / H_{i}+$ $\gamma+\alpha(i=1,2)$. Finally (41c) follows from (44) and (46).

It remains to consider the case, where $\alpha_{1}+\alpha_{2}=0$. In this case the leading coefficient with respect to $x_{1}$ of the numerator in (45) is 1 , hence

$$
\gamma\left(h_{1} G_{2}^{*}+G_{1}^{*} H_{2}\right)+G_{1}^{*} G_{2}^{*} \in K\left[x_{1}, x_{2}\right],
$$




$$
H_{1} G_{2}^{*}+G_{1}^{*} H_{2} \in K\left[x_{1}, x_{2}\right] \text {. }
$$

The leading coefficient with respect to $x_{i}$ of the left-hand side of (57) is $H_{3-i}\left(x_{3-i}\right)$, hence

$$
H_{i} \in K\left[x_{i}\right] \quad(i=1,2) .
$$

The argument leading from (48) to (51) applies mutatis mutandis and we get

$$
\begin{gathered}
G_{1}^{*}=G_{11}^{*}+\alpha H_{1}, \quad G_{2}^{*}=G_{21}^{*}-\alpha H_{2}, \\
G_{i 1}^{*} \in K\left[x_{i}\right] \quad(i=1,2), \quad \alpha \in \hat{K},
\end{gathered}
$$

Hence by (56)

$$
\begin{gathered}
\gamma\left(H_{1} G_{21}^{*}+G_{11}^{*} H_{2}\right)+G_{11}^{*} G_{21}^{*}+\alpha\left(H_{1} G_{21}^{*}-G_{11}^{*} H_{2}\right) \\
-\alpha^{2} H_{1} H_{2} \in K\left[x_{1}, x_{2}\right], \\
(\gamma+\alpha) H_{1} G_{21}^{*}+(\gamma-\alpha) G_{11}^{*} H_{2}-\alpha^{2} H_{1} H_{2} \in K\left[x_{1}, x_{2}\right] .
\end{gathered}
$$

Considering the leading coefficients with respect to $x_{1}$ and $x_{2}$ on the left-hand side of (61) we get by (60)

$$
(\gamma+\alpha) H_{1} \in K\left[x_{1}\right], \quad(\gamma-\alpha) H_{2} \in K\left[x_{2}\right],
$$

hence by (58)

$$
\gamma+\alpha \in K, \quad \gamma-\alpha \in K, \quad 2 \gamma \in K
$$

By (42) and (63) we have char $K=2$. On the other hand by (59), (61) and (62)

$$
\alpha^{2} H_{1} H_{2} \in K\left[x_{1}, x_{2}\right],
$$

hence by (53) $\alpha^{2} \in K$, by (63) $\gamma^{2} \in K$ and (41a) holds. Also (41b) holds in virtue of (43), (44), (51) and (65) with $h_{i}=\left(G_{i 1}^{*} / H_{i}\right)+\gamma+\alpha(i=1,2)$. Since $\alpha_{1}+\alpha_{2}=0$ (41c) follows from (44).

LemMa 7. If $r \in K\left(t_{1}, \ldots, t_{n}\right) \backslash\{0\}$ is of order $\rho_{i}$ with respect to $t_{i}$,

$$
\begin{gathered}
g_{i} \in K\left(x_{i}\right) \backslash K, \quad g_{i}=G_{i} / G_{i}^{*}, G_{i}, G_{i}^{*} \in K\left[x_{i}\right], \\
\left(G_{i}, G_{i}^{*}\right)=1 \quad(i=1,2, \ldots, n)
\end{gathered}
$$

$$
r\left(g_{1}, \ldots, g_{n}\right) \prod_{i=1}^{n} G_{i}^{* \rho_{t}} \in K
$$

then

$$
r\left(t_{1}, \ldots, t_{n}\right)=a \prod_{i=1}^{n}\left(t_{i}-\frac{l\left(G_{i}\right)}{l\left(G_{i}^{*}\right)}\right)^{\rho_{t}}, \quad a \in K \backslash\{0\}
$$


Proof. We proceed by induction with respect to $n$. Consider first $n=1$. Let

$$
r=a \prod_{\tau \in T}(t-\tau)^{\alpha_{\tau}}, \quad a \in K, T \subset \hat{K}, \alpha_{\tau} \neq 0 .
$$

The condition (64) gives

$$
\prod_{\tau \in T}\left(G_{1}-\tau G_{1}^{*}\right)^{\alpha_{\tau}} \in K
$$

and since

$$
\left(G_{1}-\tau G_{1}^{*}, G_{1}-\tau^{*} G_{1}^{*}\right)=1 \quad \text { for } \tau \neq \tau^{*}
$$

we have

$$
G_{1}-\tau G_{1}^{*} \in \hat{K} \quad(\tau \in T) .
$$

If $T=\varnothing(65)$ holds trivially with $\rho_{1}=0$.

If card $T \geq 2$ we get $G_{1}, G_{1}^{*} \in K$, contrary to $g_{1} \notin K$. If $T=\{\tau\}$ considering that the highest terms of $G_{1}$ and $\tau G_{1}^{*}$ cancel out, we get (65).

Assume now that the lemma is true for rational functions in $n-1$ variables, consider an $r \in K\left[t_{1}, \ldots, t_{n}\right] \backslash\{0\}$ and $g_{i}(i=1, \ldots, n)$ satisfying the assumptions of the lemma.

Let $\tau=\left(l\left(G_{n}\right) / l\left(G_{n}^{*}\right)\right)$ and put

$$
r\left(t_{1}, \ldots, t_{n}\right)=\sum_{j=0}^{r_{n}} R_{j}\left(t_{n}-\tau\right)^{r_{n}-1} / \sum_{j=0}^{r_{n}^{*}} R_{J}^{*}\left(t_{n}-\tau\right)^{r_{n}^{*}-J},
$$

where $R_{j} \in K\left[t_{1}, \ldots, t_{n-1}\right]\left(0 \leq j \leq r_{n}\right), R_{J}^{*} \in K\left[t_{1}, \ldots, t_{n-1}\right](0 \leq j \leq$ $\left.r_{n}^{*}\right), R_{0} R_{0}^{*} \neq 0$, whence $\rho_{n}=r_{n}-r_{n}^{*}$.

Since $g_{1} \in K\left(x_{i}\right) \backslash K(i=1,2, \ldots, n-1)$ we have

$$
R\left(g_{1}, \ldots, g_{n-1}\right) \neq 0 \text { if } R \in K\left[t_{1}, \ldots, t_{n-1}\right] \backslash\{0\} .
$$

Thus $R_{0} R_{0}^{*}\left(g_{1}, \ldots, g_{n-1}\right) \neq 0$ and $r\left(g_{1}, \ldots, g_{n-1}, t_{n}\right)$ is of order $\rho_{n}$ with respect to $t_{n}$. Applying the already proved case $n=1$ of our lemma to the last rational function over the field $K\left(x_{1}, \ldots, x_{n-1}\right)$ we infer from (64) that

$$
r\left(g_{1}, \ldots, g_{n-1}, t_{n}\right)=A\left(t_{n}-\tau\right)^{\rho_{n}}, \quad A \in K\left(x_{1}, \ldots, x_{n-1}\right)
$$

and

$$
A \prod_{i=1}^{n-1} G_{i}^{* \rho_{t}} \in K
$$

Now, by (66) and (68)

$$
\begin{gathered}
A=R_{0}\left(g_{1}, \ldots, g_{n-1}\right) / R_{0}^{*}\left(g_{1}, \ldots, g_{n-1}\right) \\
R,\left(g_{1}, \ldots, g_{n-1}\right)=A R_{j}^{*}\left(g_{1}, \ldots, g_{n-1}\right) \quad\left(0 \leq j \leq \min \left(r_{n}, r_{n}^{*}\right)\right)
\end{gathered}
$$




$$
\begin{array}{ll}
R_{j}\left(g_{1}, \ldots, g_{n-1}\right)=0 & \left(r_{n}^{*}<j \leq r_{n}\right), \\
R_{j}^{*}\left(g_{1}, \ldots, g_{n-1}\right)=0 & \left(r_{n}<j \leq r_{n}^{*}\right) .
\end{array}
$$

By (67), (70) and (71) $R_{j} R_{0}^{*}=R_{J}^{*} R_{0}\left(0 \leq j \leq \min \left(r_{n}, r_{n}^{*}\right)\right), R_{J}=0\left(r_{n}^{*}<\right.$ $\left.j \leq r_{n}\right), R_{j}^{*}=0\left(r_{n}<j \leq r_{n}^{*}\right)$ hence by (66)

$$
r=\frac{R_{0}}{R_{0}^{*}}\left(t_{n}-\tau\right)^{\rho_{n}}
$$

Thus $R_{0} / R_{0}^{*}$ is of order $\rho_{l}$ with respect to $x_{i}(1 \leq i<n)$. By (69), and the inductive assumption

$$
\frac{R_{0}}{R_{0}^{*}}=a \prod_{i=1}^{n-1}\left(t_{i}-\frac{l\left(G_{i}\right)}{l\left(G_{i}^{*}\right)}\right)^{\rho_{i}}, \quad a \in K
$$

and (65) follows from (72).

LEMMA 8. If $n \geq 2, g_{i} \in K\left(x_{i}\right) \backslash K, h_{i} \in K\left(t_{i}\right) \backslash K(i=1,2, \ldots, n)$, the number of pairwise nonproportional divisors of the numerator of $\sum_{i=1}^{n} h_{i}\left(g_{i}\right)$ in $K\left[x_{1}, \ldots, x_{n}\right]$ is not less than the corresponding number for the numerator of $\sum_{i=1}^{n} h_{i}$ and $K\left[t_{1}, \ldots, t_{n}\right]$.

Proof. Let for $i=1,2, \ldots, n$

$$
\begin{gathered}
g_{i}=\frac{G_{i}}{G_{i}^{*}}, \quad h_{i}=\frac{H_{i}}{H_{i}^{*}} ; \quad G_{i}, G_{i}^{*} \in K\left[x_{i}\right] ; H_{i}, H_{i}^{*} \in K\left[t_{i}\right] ; \\
\left(G_{i}, G_{i}^{*}\right)=\left(H_{i}, H_{i}^{*}\right)=1 .
\end{gathered}
$$

The numerator of $\sum_{i=1}^{n} h_{i}$ in its reduced form equals up to constant factor $\sum_{i=1}^{n} H_{i} \Pi_{j=1, j \neq i}^{n} H_{j}^{*}$ thus if $P_{1}, \ldots, P_{m}$ is a maximal set of pairwise nonproportional divisors of the said numerator we have

$$
\sum_{i=1}^{n} H_{i} \prod_{\substack{j=1 \\ j \neq i}}^{n} H_{j}^{*}=P_{k} Q_{k} \quad(k=1,2, \ldots, m),
$$

where $P_{k}, Q_{k} \in K\left[t_{1}, \ldots, t_{n}\right]$. If $p_{i k}, q_{l k}$ is the degree with respect to $x_{i}$ of $P_{k}, Q_{k}$ respectively, we get $p_{i k}+q_{i k}=\max \left(\left|H_{i}\right|,\left|H_{i}^{*}\right|\right)(i=1, \ldots, n)$. Now

$$
h_{l}\left(g_{i}\right)=\frac{H_{i}\left(G_{i}, G_{i}^{*}\right) G_{i}^{* \max \left(0,\left|H_{i}^{*}\right|-\left|H_{l}\right|\right)}}{H_{l}^{*}\left(H_{i}, G_{i}^{*}\right) G_{i}^{* \max \left(0,\left|H_{l}\right|-\left|H_{i}^{*}\right|\right)}}
$$

By (73) the numerator and the denominator on the right-hand side of (75) are coprime polynomials. Hence the numerator $N$ of $\sum_{i=1}^{n} h_{i}\left(g_{i}\right)$ is up to a 
constant factor

$$
\begin{aligned}
& \sum_{i=1}^{n} H_{i}\left(G_{i}, G_{i}^{*}\right) G_{i}^{* \max \left(0,\left|H_{\imath}^{*}\right|-\left|H_{\imath}\right|\right)} \prod_{\substack{j=1 \\
j \neq i}}^{n} H_{j}^{*}\left(G_{i}, G_{i}^{*}\right) G_{i}^{* \max \left(0,\left|H_{,}^{*}\right|-\left|H_{,}\right|\right)} \\
& =\prod_{i=1}^{n} G_{l}^{* \max \left(\left|H_{\imath}\right|,\left|H_{\imath}^{*}\right|\right)} \sum_{i=1}^{n} H_{l}\left(g_{\imath}\right) \prod_{\substack{j=1 \\
j \neq i}}^{n} H_{J}^{*}\left(g_{J}\right) \\
& =\prod_{i=1}^{n} G_{i}^{* p_{t k}+q_{t k}} P_{k}\left(g_{1}, \ldots, g_{n}\right) Q_{k}\left(g_{1}, \ldots, g_{n}\right) \\
& =\prod_{i=1}^{n} G_{i}^{* p_{t_{k}}} P_{k}\left(g_{1}, \ldots, g_{n}\right) \prod_{i=1}^{n} G_{i}^{* q_{t_{k}}} Q_{k}\left(g_{1}, \ldots, g_{n}\right) \text {. }
\end{aligned}
$$

It follows that

$$
\prod_{i=1}^{n} G_{i}^{* p_{t h}} P_{k}\left(g_{1}, \ldots, g_{n}\right) \mid N \quad(k=1,2, \ldots, m) .
$$

Assuming that for some distinct indices $k, l \leq m$

$$
\prod_{i=1}^{n} G_{i}^{* p_{i k}} P_{k}\left(g_{1}, \ldots, g_{n}\right)=c \prod_{i=1}^{n} G_{i}^{* p_{t l}} P_{l}\left(g_{1}, \ldots, g_{n}\right), \quad c \in K
$$

we could apply Lemma 7 to the rational function $P_{k} / P_{l}$. We would get by that lemma

$$
\frac{P_{k}\left(t_{1}, \ldots, t_{n}\right)}{P_{l}\left(t_{1}, \ldots, t_{n}\right)}=a \prod_{i=1}^{n}\left(t_{i}-\frac{l\left(G_{i}\right)}{l\left(G_{i}^{*}\right)}\right)^{p_{t k}-p_{l l}}, \quad a \in K .
$$

Since $P_{k} P_{l}^{-1} \notin K$ we have $p_{\imath k} \neq p_{i l}$ for at least one $i \leq n$ and without loss of generality we may assume that $p_{n k}>p_{n l}$. It follows that

$$
P_{k}\left(t_{1}, \ldots, t_{n-1}, \tau\right)=0 \text { for } \tau=l\left(G_{n}\right) / l\left(G_{n}^{*}\right)
$$

and by (74)

$$
H_{n}^{*}(\tau) \sum_{i=1}^{n} H_{1} \prod_{\substack{j=1 \\ j \neq i}}^{n} H_{j}^{*}+H_{n}(\tau) \prod_{j=1}^{n-1} H_{j}^{*}=0
$$

Since by (73) $H_{n}^{*}(\tau)=H_{n}(\tau)=0$ is impossible we have $H_{n}^{*}(\tau) \neq 0$ and

$$
\sum_{i=1}^{n-1} h_{i}+h_{n}(\tau)=0
$$

which implies $h_{1} \in K$. The obtained contradiction shows that (77) is impossible, which together with (76) completes the proof. 
COROLlaRY. Under the assumptions of Lemma 8 if $\sum_{l=1}^{n} h_{i}$ is reducible (resp. strongly reducible) over $K$ then $\sum_{i=1}^{n} h_{i}\left(g_{i}\right)$ is reducible (resp. strongly reducible) over $K$.

Proof. Reducibility (resp. strong reducibility) of $\sum_{l=1}^{n} h_{i}$ over $K$ means that the number of pairwise nonproportional divisors of its numerator in $K\left[x_{1}, \ldots, x_{n}\right]$ is at least three (resp. at least four).

Lemma 9. If $\operatorname{char} K=2, c, d \in K, \sqrt{d} \notin K, f_{i} \in K\left(x_{i}\right) \backslash K(i=$ $1,2, \ldots, n)$ and

$$
\sum_{i=1}^{n} f_{i}=\frac{c}{v^{2}+d}, \quad v \in K\left(x_{1}, \ldots, x_{n}\right)
$$

then

$$
f_{i}-c\left(f_{i}\right)=\frac{c}{g_{i}^{2}+d}, \quad g_{i} \in K\left(x_{i}\right)(i=1,2, \ldots, n)
$$

and

$$
\sum_{i=1}^{n} c\left(f_{i}\right)=0 \quad \text { or } \quad \frac{c}{g_{0}^{2}+d}, \quad g_{0} \in K .
$$

Proof. By induction on $n$. Consider first $n=1$. The equation (78) implies

$$
c\left(f_{1}\right)= \begin{cases}0 & \text { if ord } v>0 \\ \frac{c}{c(v)^{2}+d} & \text { if ord } v \leq 0\end{cases}
$$

hence

$$
f_{1}-c\left(f_{1}\right)=\frac{c}{g_{1}^{2}+d}
$$

where

$$
g_{1}= \begin{cases}v & \text { if ord } v>0 \\ \frac{d+v c(v)}{v+c(v)} & \text { if ord } v \leq 0 .\end{cases}
$$

Note that $v+c(v) \neq 0$ since by (78) $v \notin K$. Assume now that the lemma is true for $n-1$ rational functions $(n>1)$ and that (78) holds. Regarding (78) as an equation over the field $K\left(x_{n}\right)$ we get by the inductive assumptions

$$
f_{i}-c\left(f_{i}\right)=\frac{c}{g_{i}^{2}+d}, \quad g_{i} \in K\left(x_{i}, x_{n}\right)(i=1,2, \ldots, n-1),
$$


$(80)$

$$
\sum_{i=1}^{n-1} c\left(f_{i}\right)+f_{n}=0 \quad \text { or } \quad \frac{c}{\hat{g}_{0}^{2}+d}, \quad \hat{g}_{0} \in K\left(x_{n}\right) .
$$

Since the left-hand side of (79) is independent of $x_{n}$, we get $g_{i} \in K\left(x_{i}\right)$ $(i=1, \ldots, n-1)$. Since $f_{n} \notin K$ we cannot have $\sum_{i=1}^{n-1} c\left(f_{i}\right)+f_{n}=0$. Hence (80) implies by the case $n=1$ of our lemma

$$
\begin{aligned}
f_{n}-c\left(f_{n}\right) & =\frac{c}{g_{n}^{2}+d}, \quad g_{n} \in K\left(x_{n}\right) \\
\sum_{i=1}^{n} c\left(f_{i}\right) & =0 \quad \text { or } \quad \frac{c}{g_{0}^{2}+d}, \quad g_{0} \in K
\end{aligned}
$$

and the inductive proof is complete.

LEMMA 10. If $\operatorname{char} K=2, f_{i} \in K\left(x_{i}\right)(i=1,2, \ldots, n)$,

$$
\sum_{i=1}^{n} f_{l}=b v^{2}, \quad b \in K, v \in K\left(x_{1}, \ldots, x_{n}\right)
$$

then

$$
f_{i}-c\left(f_{i}\right)=b g_{i}^{2}, \quad g_{i} \in K\left(x_{i}\right)(i=1,2, \ldots, n)
$$

and

$$
\sum_{i=1}^{n} c\left(f_{i}\right)=b g_{0}^{2}, \quad g_{0} \in K .
$$

Proof. By induction on $n$.

For $n=1$ the equation

$$
f_{1}=b v^{2} ; \quad v \in K\left(x_{1}\right)
$$

implies

$$
c\left(f_{1}\right)=b c(v)^{2}
$$

hence

$$
f_{1}-c\left(f_{1}\right)=b(v-c(v))^{2},
$$

which proves the assertion.

Assume now that the lemma is true for $n-1$ rational functions $(n \geq 2)$ and consider the equation

$$
\sum_{i=1}^{n} f_{i}=b v^{2}
$$


over the field $K\left(x_{n}\right)$. Applying the inductive assumption we infer that

$$
f_{i}-c\left(f_{i}\right)=b g_{i}^{2}, \quad g_{i} \in K\left(x_{i}, x_{n}\right)(i=1, \ldots, n-1)
$$

and

$$
\sum_{i=1}^{n-1} c\left(f_{i}\right)+f_{n}=b \hat{g}_{0}^{2}, \quad \hat{g}_{0} \in K\left(x_{n}\right) .
$$

Since the left-hand side of the first equation is independent of $x_{n}$ we have $g_{i} \in K\left(x_{i}\right)(i=1, \ldots, n-1)$. The second equation gives in virtue of the case $n=1$ of our lemma

$$
\begin{gathered}
f_{n}-c\left(f_{n}\right)=b g_{n}^{2}, \quad g_{n} \in K\left(x_{n}\right), \\
\sum_{i=1}^{n} c\left(f_{i}\right)=b g_{0}^{2}, \quad g_{0} \in K
\end{gathered}
$$

and the inductive proof is complete.

Lemma 11. If $n \geq 3, f_{i} \in K\left(x_{l}\right) \backslash K(i=1,2, \ldots, n)$ and $\sum_{i=1}^{n} f_{i}$ is reducible (resp. strongly reducible) over $K$ then there exist an additive polynomial $\hat{L} \in \hat{K}[z]$ and rational functions $\hat{g}_{i} \in \hat{K}\left(x_{i}\right)(i=1,2)$ such that

$$
f_{l}-c\left(f_{i}\right)=\hat{L}\left(\hat{g}_{\imath}\right) \quad(i=1,2)
$$

and either

$$
\begin{gathered}
\hat{L} \in K[z], \quad \hat{g}_{i} \in K\left(x_{i}\right) \quad(i=1,2) \\
\hat{L}(z)+c\left(f_{1}\right)+c\left(f_{2}\right)+\sum_{i=3}^{n} f_{i} \text { is reducible }
\end{gathered}
$$

(resp. strongly reducible) over $K$ or $\operatorname{char} K=2$ and there exist $\gamma, \delta \in \hat{K}, h_{0} \in K, h_{i} \in K\left(x_{i}\right)(i=1,2)$ satisfying

$$
\begin{gathered}
\gamma \notin K, \quad \gamma^{2} \in K, \quad \delta=0 \text { or } \frac{1}{h_{0}+\gamma} ; \\
\hat{L}\left(\frac{1}{t-\gamma}-\delta\right) \in K(t), \quad \hat{g}_{i}=\frac{1}{h_{i}+\gamma} \quad(i=1,2) ; \\
\hat{L}\left(\frac{1}{t-\gamma}-\delta\right)+c\left(f_{1}\right)+c\left(f_{2}\right)+\sum_{i=3}^{n} f_{i} \text { is reducible }
\end{gathered}
$$

(resp. strongly reducible) over $K$. 
Proof. Reducibility (resp. strongly reducibility) of $\sum_{l=1}^{n} f_{l}$ over $K$ implies by Lemma 1 with $f=f_{1}+f_{2}, v=t+\sum_{i=3}^{n} f_{t}$ that

$$
\begin{gathered}
f_{1}+f_{2}=h(g) \\
h \in K(t), \quad g \in K\left(x_{1}, x_{2}\right)
\end{gathered}
$$

and

$$
h+\sum_{i=3}^{n} f_{l} \text { is reducible (resp. strongly reducible) over } K .
$$

By Lemma 4 (88) implies either

$$
h=H(t), \quad g=g_{1}+g_{2}
$$

or

$$
h=H\left(\frac{1}{t-\gamma}\right), \quad g=\gamma+\frac{1}{g_{1}+g_{2}},
$$

where $H \in \hat{K}[z], \gamma \in \hat{K}, g_{\imath} \in \hat{K}\left(x_{l}\right)(i=1,2)$. In both cases

$$
f_{1}+f_{2}=H\left(g_{1}+g_{2}\right)
$$

hence by Lemma 5

$$
H-H(0) \text { is an additive polynomial }
$$

and

$$
\begin{gathered}
f_{t}-c\left(f_{l}\right)=H\left(g_{\imath}-c\left(g_{\imath}\right)\right)-H(0) \quad(i=1,2), \\
c\left(f_{l}\right)+c\left(f_{2}\right)=H\left(c\left(g_{1}\right)+c\left(g_{2}\right)\right) .
\end{gathered}
$$

Put

(96) $\hat{L}=H-H(0), \quad \hat{g}_{\imath}=g_{\imath}-c\left(g_{i}\right) \quad(i=1,2), \quad \delta=c\left(g_{1}\right)+c\left(g_{2}\right)$.

By (93) $\hat{L}$ is an additive polynomial and by (94) we have (81). Moreover by (95)

$$
H(z)=\hat{L}(z-\delta)+c\left(f_{1}\right)+c\left(f_{2}\right) .
$$

In case (90) we get from (88) both (82) and $\delta \in K$, hence (83) follows from (89) and (97).

In case (91) we get from (88), (89) and (97)

$$
\begin{gathered}
\hat{L}\left(\frac{1}{t-\gamma}-\delta\right) \in K(t), \\
\hat{L}\left(\frac{1}{t-\gamma}-\delta\right)+c\left(f_{1}\right)+c\left(f_{2}\right)+\sum_{l=3}^{n} f_{l} \text { is reducible } \\
\text { (resp. strongly reducible) over } K .
\end{gathered}
$$


On the other hand by (88) and Lemma 6 either

$$
\gamma \in K, \quad(82) \text { holds }
$$

or for suitable $h_{0} \in K, h_{i} \in K\left(x_{l}\right)(i=1,2)$

$$
\text { (84) holds and } \quad \hat{g}_{\imath}=\frac{1}{h_{i}+\gamma} \quad(i=1,2) .
$$

In the former case in virtue of (99) and Corollary to Lemma 8 we have (83). In the latter case by (98) we have (85) and (86) is identical with (99).

LEMMA 12. If $n \geq 3, f_{i} \in K\left(x_{i}\right) \backslash K(i=1,2, \ldots, n), \max _{1 \leq i \leq n}$ ord $f_{i}$ $>0$ and $\sum_{i=1}^{n} f_{i}$ is reducible (resp. strongly reducible) over $K$ then there exist an additive polynomial $L \in K[z]$ and rational functions $h_{i} \in K\left(x_{i}\right)(i=$ $1, \ldots, n)$ such that

$$
f_{i}-c\left(f_{i}\right)=L\left(g_{i}\right) \quad(i=1,2, \ldots, n)
$$

and

(101) $L(z)+\sum_{i=1}^{n} c\left(f_{l}\right)$ is reducible (resp. strongly reducible) over $K$.

Proof. We assume without loss of generality that

$$
\text { ord } f_{1}>0
$$

and proceed by induction with respect to $n$. Consider first $n=3$. By Lemma 11 there exist an additive polynomial $\hat{L} \in \hat{K}[z]$ and rational functions $\hat{g}_{i} \in \hat{K}\left(x_{i}\right)(i=1,2)$ satisfying (81) and either (82), (83) or (84)-(86). In the latter case by (85) ord $\hat{g}_{1} \leq 0$ hence by (81) ord $f_{1} \leq 0$ contrary to (102). Thus we have (81)-(83). Substituting in (83) $z=x_{1}+x_{2}$ we infer that $\hat{L}\left(x_{1}\right)+\hat{L}\left(x_{2}\right)+c\left(f_{1}\right)+c\left(f_{2}\right)+f_{3}$ is reducible (resp. strongly reducible) over $K$.

Applying to the above sum the result already established but reversing the roles of $x_{2}$ and $x_{3}$ we infer in analogy to (81)-(83) the existence of an additive polynomial $L \in K[z]$ and rational functions $h_{i} \in K\left(x_{i}\right)(i=1,3)$ such that

$$
\begin{array}{r}
\hat{L}\left(x_{1}\right)=L\left(h_{1}\right) \\
f_{3}-c\left(f_{3}\right)=L\left(h_{3}\right) \\
(104)
\end{array}
$$


It follows from (81) and (103), (104) that (100) is satisfied with $g_{\imath}=h_{1}\left(\hat{g}_{i}\right)$ $(i=1,2), g_{3}=h_{3}$.

On the other hand by (103) and (105)

$$
L\left(z+h_{1}\left(x_{2}\right)\right)+\sum_{i=1}^{3} c\left(f_{i}\right) \text { is reducible }
$$

(resp. strongly reducible) over $K$

hence (101) holds.

Now assume that the Lemma holds for sums of $n-1$ rational functions $(n \geq 4)$ and suppose that $f_{i} \in K\left[x_{i}\right] \backslash K(i=1,2, \ldots, n), \sum_{i=1}^{n} f_{i}$ is reducible (resp. strongly reducible) over $K$ and (102) holds.

Applying as before Lemma 11 we find that (81)-(83) hold for suitable $\hat{L}$ and $\hat{g}_{i}(i=1,2)$. Applying to (83) the inductive assumption we infer the existence of an additive polynomial $L \in K[t]$ and rational functions $h \in K(z), h_{i} \in K\left(x_{i}\right)(i=3, \ldots, n)$ such that

$$
\begin{gathered}
\hat{L}(z)=L(h), \\
f_{i}-c\left(f_{i}\right)=L\left(h_{i}\right) \quad(i=3, \ldots, n),
\end{gathered}
$$

(108) $L+\sum_{i=1}^{n} c\left(f_{l}\right)$ is reducible (resp. strongly reducible) over $K$.

It follows from (81) and (106), (107) that (100) is satisfied with

$$
g_{i}=h\left(\hat{g}_{i}\right) \quad(i=1,2), \quad g_{i}=h_{i} \quad(i=3, \ldots, n) .
$$

On the other hand (101) is identical with (108). The inductive proof is complete.

Proof of the theorem. The condition (i) or (ii) or (iii) is necessary. Assume w.l.o.g.

$$
\text { ord } f_{1} \geq \text { ord } f_{\imath} \quad(i=1,2, \ldots, n) .
$$

Reducibility of $\sum_{i=1}^{n} f_{i}$ implies by Lemma 11 the existence of an additive polynomial $\hat{L} \in \hat{K}[z]$ and of rational functions $\hat{g}_{i} \in \hat{K}\left(x_{i}\right)(i=$ $1,2)$ satisfying (81) and either (82), (83) (Case A) or for char $K=2$ and suitable $\gamma, \delta \in \hat{K}, h_{0} \in K\left(x_{i}\right)(i=1,2)(84)-(86)$ (Case B).

We shall consider these cases successively.

A. Substituting in (83) $z=x_{1}+x_{2}$ we infer that

$$
\hat{L}\left(x_{1}\right)+\hat{L}\left(x_{2}\right)+c\left(f_{1}\right)+c\left(f_{2}\right)+\sum_{i=3}^{n} f_{i} \text { is reducible over } K \text {. }
$$


By Lemma 12 there exist an additive polynomial $L \in K[z]$ and rational functions

$$
g \in K\left(x_{1}\right), \quad g_{i} \in K\left(x_{i}\right) \quad(i=3, \ldots, n)
$$

such that

$$
\begin{gathered}
\hat{L}\left(x_{1}\right)=L(g), \\
f_{i}-c\left(f_{i}\right)=L\left(g_{i}\right) \quad(i=3, \ldots, n), \\
L(z)+\sum_{i=1}^{n} c\left(f_{i}\right) \text { is reducible over } K .
\end{gathered}
$$

It follows from (81) and (110) that

$$
f_{i}-c\left(f_{i}\right)=L\left(g\left(\hat{g}_{i}\right)\right) \quad(i=1,2)
$$

thus condition (i) is satisfied with $g_{i}=g\left(\hat{g}_{i}\right)(i=1,2)$.

B. Let $\tilde{K}=K(\gamma)$. By (84) $\tilde{K}$ is a quadratic inseparable extension of $K$, by (84) and (85)

$$
\delta \in \tilde{K}, \quad \hat{L} \in \tilde{K}[t], \quad \hat{g}_{i} \in \tilde{K}\left(x_{\imath}\right)(i=1,2) .
$$

By (85) ord $\hat{g}_{1} \leq 0$ hence by (81) ord $f_{1} \leq 0$ and by (109)

$$
\text { ord } f_{i} \leq 0 \quad(i=1,2, \ldots, n) .
$$

We distinguish two cases.

$$
\hat{L}\left(\frac{1}{t-\gamma}-\delta\right)+c\left(f_{1}\right)+c\left(f_{2}\right)+\sum_{i=3}^{n} f_{i}
$$

is strongly reducible over $\tilde{K}$,

$$
\hat{L}\left(\frac{1}{t-\gamma}-\delta\right)+c\left(f_{1}\right)+c\left(f_{2}\right)+\sum_{i=3}^{n} f_{i}
$$

is not strongly reducible over $\tilde{K}$.

B1. By (114) and Corollary to Lemma 8

$$
\hat{L}(z)+c\left(f_{1}\right)+c\left(f_{2}\right)+\sum_{i=3}^{n} f_{i} \text { is strongly reducible over } \tilde{K}
$$

and on substituting $z=x_{1}+x_{2}$

$$
\hat{L}\left(x_{1}\right)+\hat{L}\left(x_{2}\right)+c\left(f_{1}\right)+c\left(f_{2}\right)+\sum_{i=3}^{n} f_{i} \text { is strongly reducible over } \tilde{K} \text {. }
$$


By Lemma 12 there exist an additive polynomial $L \in \tilde{K}[z]$ and rational functions $g \in \tilde{K}\left(x_{1}\right), g_{\imath} \in \tilde{K}\left(x_{\imath}\right)(i=3, \ldots, n)$ such that (110) and (111) hold and

$$
L(z)+\sum_{i=1}^{n} c\left(f_{i}\right) \text { is strongly reducible over } \tilde{K} \text {. }
$$

(81) and (110) imply (112) thus in view of (113)-(114) and (115) condition (iii) is satisfied with $g_{\imath}=g\left(\hat{g}_{\imath}\right)(i=1,2)$.

B.2 Let $N$ be the numerator (defined only up to a constant factor) of

$$
\hat{L}\left(\frac{1}{t-\gamma}-\delta\right)+c\left(f_{1}\right)+c\left(f_{2}\right)+\sum_{i=3}^{n} f_{l} .
$$

Since $N$ is reducible over $K$ by (86) and not strongly reducible over $\tilde{K}$ we have $|\hat{L}|>1$,

$$
N=a M^{2} \text { for some } a \in K, M \in K\left[t, x_{3}, \ldots, x_{n}\right] .
$$

Since by (114) $\hat{L}$ is an additive polynomial over $\tilde{K}$ and char $\tilde{K}=2$ we have

$$
\hat{L}=\sum_{i=0}^{k} c_{t} z^{z^{i}}, \quad c_{t} \in \tilde{K}(i=0,1, \ldots, k), c_{k} \neq 0 .
$$

Consider first $k \quad 1$ and let

$$
\begin{aligned}
\hat{L}(\delta)+c\left(f_{1}\right)+c\left(f_{2}\right)+ & \sum_{i=3}^{n} f_{l}=\frac{F}{F^{*}}, \\
& F, F^{*} \in \tilde{K}\left[x_{3}, \ldots, x_{n}\right],\left(F, F^{*}\right)=1 .
\end{aligned}
$$

We get for a suitable $\rho \in \tilde{K}$

$$
\rho N=(t-\gamma)^{2} F+c_{0}(t-\gamma) F^{*}+c_{1} F^{*} .
$$

By (116) $t$ may occur in $N$ in even powers only, hence

$$
c_{0}=0 \text {. }
$$

The coefficients of $t^{2 i}(i=0,1)$ in $N$ must be of the form $a M_{l}^{2}, M_{l} \in$ $K\left[x_{3}, \ldots, x_{n}\right]$, i.e.

$$
\gamma^{2} F+c_{1} F^{*}=\rho a M_{0}^{2}, \quad F=\rho a M_{1}^{2} .
$$

Since $f_{t} \in K\left(x_{t}\right) \backslash K(i=3, \ldots, n)$ we have by (118) $F \neq 0$, thus

$$
\gamma^{2}+c_{1} \frac{F^{*}}{F}=\left(\frac{M_{0}}{M_{1}}\right)^{2}, \quad \frac{F}{F^{*}}=\frac{c_{1}}{\left(M_{0} / M_{1}\right)^{2}+\gamma^{2}} .
$$

By (81), (85), (117) and (119)

$$
f_{l}-c\left(f_{l}\right)=c_{1} \hat{g}_{l}^{2}=\frac{c_{1}}{h_{i}^{2}+\gamma^{2}} \quad(i=1,2)
$$

hence $c_{1} \in K$. 
By (118), (120) and Lemma 9 applied with $d=\gamma^{2}$ we get

$$
\begin{gathered}
f_{l}-c\left(f_{i}\right)=\frac{c_{1}}{g_{i}^{2}+\gamma^{2}}, \quad g_{i} \in K\left(x_{i}\right) \quad(i=3, \ldots, n), \\
\hat{L}(\delta)+\sum_{i=1}^{n} c\left(f_{l}\right)=0 \quad \text { or } \quad \frac{c_{1}}{g_{0}^{2}+\gamma^{2}}, \quad g_{0} \in K .
\end{gathered}
$$

It follows from (84), (117) and (119) that

$$
\hat{L}(\delta)=0 \quad \text { or } \quad \frac{c_{1}}{h_{0}^{2}+\gamma^{2}}
$$

thus

$$
\sum_{i=1}^{n} c\left(f_{i}\right)=0 \quad \text { or } \quad \frac{c_{1}}{g_{0}^{2}+\gamma^{2}} \text { or } \frac{c_{1}}{h_{0}^{2}+\gamma^{2}} \text { or } \frac{c_{1}}{g_{0}^{2}+\gamma^{2}}+\frac{c_{1}}{h_{0}^{2}+\gamma^{2}} .
$$

However

$$
\frac{c_{1}}{g_{0}^{2}+\gamma^{2}}+\frac{c_{1}}{h_{0}^{2}+\gamma^{2}}= \begin{cases}0 & \text { if } g_{0}=h_{0}, \\ \frac{c_{1}}{\left(g_{0} h_{0} /\left(g_{0}+h_{0}\right)\right)^{2}+\gamma^{2}} & \text { if } g_{0} \neq h_{0} .\end{cases}
$$

Therefore (ii) holds with $c=c_{1}, d=\gamma^{2}, g_{0}$ replaced if necessary by $h_{0}$ or $g_{0} h_{0} /\left(g_{0}+h_{0}\right)$ and $g_{i}=h_{i}(i=1,2)$.

Consider now $k>1$ and let again (118) hold. We get for a suitable $\rho \in \tilde{K}$

$$
\rho N=(t-\gamma)^{2^{k}} F+\sum_{i=0}^{k} c_{i}(t-\gamma)^{2^{k}-2^{i}} F^{*} .
$$

The leading coefficient of $N$ with respect to $t$ is $\rho^{-1} F$ hence by (116)

$$
F=\rho a M_{0}^{2}, \quad M_{0} \in K\left[x_{3}, \ldots, x_{n}\right] .
$$

It follows that

$$
\sum_{i=0}^{k} c_{t}(t-\gamma)^{2^{k}-2^{\prime}} F^{*}=\rho a\left(M-(t-\gamma)^{2^{k-1}} M_{0}\right)^{2}
$$

and since $(t-\gamma)^{2^{k-1}} \in K[t]$ we have for a suitable $b \in \tilde{K}, M_{1} \in$ $K\left[x_{3}, \ldots, x_{n}\right]$ and $M_{2} \in K[t]$.

$$
\sum_{i=0}^{k} c_{i}(t-\gamma)^{2^{k}-2^{t}}=b M_{2}^{2}, \quad F^{*}=\rho a b^{-1} M_{1}^{2} .
$$

Hence by (117)

$$
\hat{L}=\sum_{i=0}^{k} c_{i} z^{2^{i}}=b z^{2^{k}} M_{2}\left(\frac{1}{z}+\gamma\right)^{2}
$$


and by (81) and (85)

$$
\begin{aligned}
f_{i}-c\left(f_{i}\right) & =b\left(\frac{1}{h_{i}+\gamma}\right)^{2^{k}} M_{2}\left(h_{l}\right)^{2} \\
& =b\left(\left(\frac{1}{h_{\imath}^{2}+\gamma^{2}}\right)^{2^{k-2}} M_{2}\left(h_{i}\right)\right)^{2} \quad(i=1,2) .
\end{aligned}
$$

It follows that $b \in K$. Furthermore, by (121) and (122)

$$
\hat{L}(\delta)+c\left(f_{1}\right)+c\left(f_{2}\right)+\sum_{i=3}^{n} f_{i}=b\left(\frac{M_{0}}{M_{1}}\right)^{2},
$$

hence by Lemma 10

$$
\begin{gathered}
f_{l}-c\left(f_{i}\right)=b g_{i}^{2}, \quad g_{i} \in K\left(x_{l}\right)(i=3, \ldots, n), \\
\hat{L}(\delta)+\sum_{i=1}^{n} c\left(f_{l}\right)=b g_{0}^{2}, \quad g_{0} \in K .
\end{gathered}
$$

It follows from (84) and (123) that

$$
\hat{L}(\delta)=0 \quad \text { or } \quad b\left(\frac{1}{h_{0}^{2}+\gamma^{2}}\right)^{2^{k-1}} M_{2}\left(h_{0}\right)^{2}
$$

hence

$$
\sum_{l=1}^{n} c\left(f_{l}\right)=b g_{0}^{2} \quad \text { or } \quad b\left(g_{0} \frac{M_{2}\left(h_{0}\right)}{\left(h_{0}^{2}+\gamma^{2}\right)^{2^{k-2}}}\right)^{2} \text {. }
$$

The condition (i) is satisfied with $L=b z^{2}$.

The conditions (i), (ii), (iii) are sufficient.

(i) If (2) holds then also

$$
L\left(\sum_{l=1}^{n} t_{l}\right)+\sum_{i=1}^{n} c\left(f_{i}\right)=\sum_{i=1}^{n} L\left(t_{l}\right)+\sum_{l=1}^{n} c\left(f_{l}\right) \text { is reducible over } K
$$

In virtue of Corollary to Lemma 8

$$
\sum_{i=1}^{n} L\left(g_{i}\right)+\sum_{l=1}^{n} c\left(f_{i}\right) \text { is reducible over } K
$$

and by (1) the above sum equals $\sum_{i=1}^{n} f_{i}$.

(ii) In virtue of Corollary to Lemma 8 it is enough to show that the rational functions

$$
f_{n}\left(x_{1}, \ldots, x_{n}\right)=\sum_{i=1}^{n} \frac{1}{x_{i}^{2}+d}
$$


and $f_{n+1}\left(x_{1}, \ldots, x_{n}, g_{0}\right)$ are reducible over $K$ for every $g_{0} \in K$ and every $d \in K$ such that $\sqrt{d} \notin K$.

The numerator of $f_{n}$ equals up to a constant factor

$$
\begin{aligned}
N_{n}\left(x_{1}, \ldots, x_{n}\right) & =\sum_{i=1}^{n} \sum_{k=0}^{n-1} d^{k} \tau_{n-1-k}\left(x_{1}^{2}, \ldots, x_{1-1}^{2}, x_{l+1}^{2}, \ldots, x_{n}^{2}\right) \\
& =\sum_{k=0}^{n-1} \sum_{i=1}^{n} \tau_{n-1-k}\left(x_{1}^{2}, \ldots, x_{l-1}^{2}, x_{l+1}^{2}, \ldots, x_{n}^{2}\right),
\end{aligned}
$$

where $\tau_{j}$ is the $j$ th fundamental symmetric function. We have by a simple counting argument

$$
\sum_{t=1}^{n} \tau_{n-1-k}\left(x_{1}^{2}, \ldots, x_{i-1}^{2}, x_{t+1}^{2}, \ldots, x_{n}^{2}\right)=(k+1) \tau_{n-1-k}\left(x_{1}^{2}, \ldots, x_{n}^{2}\right)
$$

for $k$ odd the right hand vanishes (char $K=2$ ), hence

$$
\begin{aligned}
N_{n}\left(x_{1}, \ldots, x_{n}\right) & =\sum_{\substack{k=0 \\
k \text { even }}}^{n-1} d^{k} \tau_{n-1-k}\left(x_{1}^{2}, \ldots, x_{n}^{2}\right) \\
& =\left(\sum_{\substack{k=0 \\
k \text { even }}}^{n-1} d^{k / 2} \tau_{n-1-k}\left(x_{1}, \ldots, x_{n}\right)\right)^{2},
\end{aligned}
$$

which proves that $f_{n}\left(x_{1}, \ldots, x_{n}\right)$ is reducible over $K$. The numerator of $f_{n+1}\left(x_{1}, \ldots, x_{n}, g_{n}\right)$ equals up to a constant factor

$$
N_{n+1}\left(x_{1}, \ldots, x_{n}, g_{0}\right)=\left(\sum_{k=0}^{n} d^{k / 2} \tau_{n-k}\left(x_{1}, \ldots, x_{n}, g_{0}\right)\right)^{2}
$$

hence $f_{n+1}\left(x_{1}, \ldots, x_{n}, g_{0}\right)$ is reducible over $K$.

(iii) In this case $L(z)+\sum_{l=1}^{n} c\left(f_{i}\right)$ is strongly reducible over $\tilde{K}$, hence

$$
L\left(\sum_{i=1}^{n} t_{i}\right)+\sum_{i=1}^{n} c\left(f_{i}\right)=\sum_{i=1}^{n} L\left(t_{i}\right)+\sum_{i=1}^{n} c\left(f_{i}\right)
$$

is strongly reducible over $\tilde{K}$. By Corollary to Lemma 8

$$
\sum_{l=1}^{n} L\left(g_{l}\right)+\sum_{i=1}^{n} c\left(f_{l}\right)
$$

is also strongly reducible over $\tilde{K}$. By (1) the above sum equals $\sum_{i=1}^{n} f_{i}$ and denoting its numerator by $N$ we have

$$
N=P Q ; \quad P, Q \in \tilde{K}\left[x_{1}, \ldots, x_{n}\right] \backslash \tilde{K}, P Q^{-1} \notin \tilde{K} .
$$

It follows that

$$
N^{2}=P^{2} Q^{2}
$$


and since $\tilde{K}$ is a quadratic inseparable extension of $K$ we have

$$
P^{2}, Q^{2} \in K\left[x_{1}, \ldots, x_{n}\right] \backslash K \text {. }
$$

If $N$ were irreducible over $K(125)$ would imply $P^{2} Q^{-2} \in K$ contrary to (124).

Proof of Corollary 1. If char $K=0$ all nonzero additive polynomials are of degree 1 , hence $L(z)+\sum_{l=1}^{n} c\left(f_{t}\right)$ is irreducible over $K$.

Proof of Corollary 2. If char $K=p$ and $K$ is algebraically closed then as observed by Tverberg [6] every additive polynomial in $K[z]$ of degree greater than 1 is of the form $L_{0}^{p}+c L_{0}, L_{0} \in K[z], c \in K$. Since additive polynomials of degree 1 do not satisfy (2) we have by (1) $f_{t}-c\left(f_{i}\right)=$ $L_{0}\left(g_{l}\right)^{p}+c L_{0}\left(g_{l}\right)(i=1,2, \ldots, n)$.

We can also find elements $a_{\imath} \in K$ such that

$$
c\left(f_{t}\right)=a_{i}^{p}+c a_{i} \quad(i=1,2, \ldots, n) .
$$

Hence

$$
f_{i}=k_{i}^{p}+c h_{i} \quad(i=1,2, \ldots, n)
$$

with $h_{\imath}=L_{0}\left(g_{i}\right)+a_{i} \in K\left(x_{i}\right)$.

Proof of Corollary 3. If char $K \neq 2$ or $K$ is perfect there are no quadratic inseparable extensions of $K$, hence conditions (ii), (iii) are never satisfied.

EXAMPLE. Let $K$ be a field of characteristic 2 that is not perfect and let $d$ be an element of $K$, such that $\sqrt{d} \notin K$. Take

$$
f_{l}=\frac{x_{i}}{x_{i}^{2}+d} \quad(i=1,2, \ldots, n) .
$$

We have $c\left(f_{1}\right)=0$ and $f_{1}$ cannot be represented in the form $L\left(g_{1}\right)$, with $L \in K[z], g_{1} \in K\left(x_{1}\right)$ for any polynomial $L$ of degree $l>1$. Indeed in that case $x_{1}^{2}+d=c G^{l}, c \in K, G \in K\left[x_{1}\right]$, hence $l=2, \sqrt{d} \in K$. Also $f_{1} \neq c /\left(g_{1}^{2}+d_{1}\right)$, where $c, d_{1} \in K, g \in K\left(x_{1}\right)$, since the order of the left-hand side is -1 and that of the right-hand side is even. On the other hand

$$
\frac{x}{x^{2}+d}=\sqrt{d}\left(\frac{1}{x+\sqrt{d}}\right)^{2}+\frac{1}{x+\sqrt{d}}
$$


and the additive polynomial $\sqrt{d} z^{2}+z$ is strongly reducible over $K(\sqrt{d})$, thus $f_{i}$ satisfy condition (iii). We note that

$$
\sum_{i=1}^{n} f_{i}=\frac{\left(\sum_{j=0}^{1 n} d^{(n-j) / 2} \tau_{j}\left(x_{1}, \ldots, x_{n}\right)\right) \cdot\left(\sum_{j=0}^{2 n} d^{(n-j-1) / 2} \tau_{j}\left(x_{1}, \ldots, x_{n}\right)\right)}{\prod_{i=1}^{n}\left(x_{i}^{2}+d\right)}
$$

where $\tau_{0}=1, \tau_{j}$ is the $j$ th fundamental symmetric function, $\Sigma^{1}$ is taken over $j \equiv n(\bmod 2)$ and $\sum^{2}$ over $j \not \equiv n \bmod 2$.

\section{REFERENCES}

[0] M. Fried, The field of definition of function fields and a problem in the reducibility of polynomials in two variables, Illinois J. Math., 17 (1973), 128-144.

[1] M. Fried and R. E. MacRae, On curves with separated variables, Math. Ann., 180 (1969), 220-226.

[2] J. Igusa, On a theorem of Lueroth, Mem. Coll. Sci. Univ. Kyoto, Ser. A. Math., 26 (1951), 251-259.

[3] A. Schinzel, Some unsolved problems on polynomials in the book Neki nerešeni problemi u matematici, Matematička Biblioteka, 25 Beograd (1963), 63-70.

[4] _ Selected Topics on Polynomials, The University of Michigan Press, 1982.

[5] H. Tverberg, A remark on Ehrenfeucht's criterion for irreducibility of polynomials, Prace Mat., 8 (1964), 117-118.

[6] On the irreducibility of polynomials $f(x)+g(y)+h(z)$, Quart. J. Math. Oxford Ser. (2), 17 (1966), 364-366.

Received May 2, 1984 and in revised form September 10, 1984.

Mathematics Institute PAN

P. O. Box 137

00-950 Warszawa, Poland 
